\title{
Gastric cancer treatment in Japan: 2008 annual report of the JGCA nationwide registry
}

\author{
Yoh Isobe $\cdot$ Atsushi Nashimoto $\cdot$ Kohei Akazawa - Ichiro Oda Kenichi Hayashi \\ Isao Miyashiro $\cdot$ Hitoshi Katai $\cdot$ Shunichi Tsujitani · Yasuhiro Kodera • \\ Yasuyuki Seto $\cdot$ Michio Kaminishi
}

Received: 2 October 2010/Accepted: 19 July 2011/Published online: 7 September 2011

(c) The Author(s) 2011. This article is published with open access at Springerlink.com

\begin{abstract}
The Japanese Gastric Cancer Association (JGCA) started a new nationwide gastric cancer registry in 2008. Approximately 50 data items, including surgical procedures, pathological diagnoses, and survival outcomes, for 12004 patients with primary gastric cancer treated in 2001 were collected retrospectively from 187 participating hospitals. Data were entered into the JGCA database according to the JGCA Classification of gastric carcinoma, 13th edition and the International Union Against Cancer (UICC) TNM Classification of malignant tumors, 5th edition by using an electronic data collecting system. Finally,
\end{abstract}

All the authors belong to the Registration Committee of the Japanese Gastric Cancer Association.

Y. Isobe $(\square)$

Department of Surgery, National Hospital Organization Tokyo

Medical Center, 2-5-1 Higashigaoka, Meguro-ku,

Tokyo 152-8902, Japan

e-mail: isobey@mb.infoweb.ne.jp

A. Nashimoto

Department of Surgery, Niigata Cancer Center Hospital,

Niigata, Japan

K. Akazawa

Department of Medical Informatics, Niigata University Medical and Dental Hospital, Niigata, Japan

I. Oda

Endoscopy Division, National Cancer Center Hospital,

Tokyo, Japan

K. Hayashi

Department of Surgery, Yamagata Prefectural Kahoku Hospital,

Yamagata, Japan

I. Miyashiro

Department of Surgery, Osaka Medical Center for Cancer

and Cardiovascular Diseases, Osaka, Japan data of 11261 patients with gastric resection were analyzed. The 5-year follow-up rate was $83.5 \%$. The direct death rate was $0.6 \%$. TNM 5-year survival rates (5YSRs)/JGCA 5YSRs were $91.8 / 91.9 \%$ for stage IA, $84.6 / 85.1 \%$ for stage IB, $70.5 / 73.1 \%$ for stage II, $46.6 / 51.0 \%$ for stage IIIA, $29.9 / 33.4 \%$ for stage IIIB, and $16.6 / 15.8 \%$ for stage IV. The proportion of patients more than 80 years old was $7.0 \%$, and their 5YSR was $48.7 \%$. Compared to the JGCA archived data, though the follow-up rate needs to be improved, these data suggest that the postoperative results of patients with primary gastric carcinoma have improved in those with advanced disease and in the aged population in Japan.

\section{H. Katai}

Department of Surgery, National Cancer Center Hospital, Tokyo, Japan

S. Tsujitani

Department of Surgery and Science, Graduate School of Medical

Science, Kyushu University, Fukuoka, Japan

Y. Kodera

Department of Surgery, Nagoya University School of Medicine,

Nagoya, Japan

Y. Seto

Department of Gastrointestinal Surgery, Graduate School

of Medicine, University of Tokyo, Tokyo, Japan

M. Kaminishi

Department of Surgery, Showa General Hospital,

Tokyo, Japan 
Keywords Gastric cancer · Registry - Survival rate . Japan

\section{Introduction}

From 1998, the Japanese Gastric Cancer Association (JGCA) began conducting a nationwide gastric cancer registration project by using electronic data collecting systems. Detailed survival analyses of 8851 patients with primary gastric cancer treated in 1991 were reported in 2006 [1]. However, this nationwide registry was suspended because of several issues such as the operation of the Act Concerning Protection of Personal Information, revision of the JGCA classification for gastric cancer, and rapid changes in the information technology (IT) environment at the member hospitals. After a period of 10 years in which the program was inactive, the registration committee of the JGCA started a new registration program to collect anonymized data simply, correctly, and quickly, in 2008 [2, 3]. Based on this program, we investigated the survival outcomes of patients with primary gastric cancer treated in 2001.

\section{Subjects, materials, and methods}

In the 2008 JGCA nationwide registration program, approximately 50 data items, including surgical procedures, pathological diagnoses, and prognoses, for patients with primary gastric carcinoma surgically treated in 2001 were collected retrospectively in 2008 by using custommade software. This software could be downloaded from the JGCA website. The JGCA member hospitals could participate in this project voluntarily.
The registration data of this system are listed in Table 1. Definition and documentation of the items were based on the Japanese (JGCA) Classification of gastric carcinoma, 13th edition [4, 5] and the International Union Against Cancer (UICC) TNM Classification of malignant tumors, 5th edition [6]. These two classifications were not compatible with each other and items could not be converted automatically. The JGCA T-category was identical to the TNM classification. On the other hand, in the JGCA classification, peritoneal metastasis and liver metastasis were individually recorded as $\mathrm{P}$ - and $\mathrm{H}$-categories, both of which could be translated into the M-category in the TNM classification. Intraoperative peritoneal washing cytology (CY) was an independent category in the JGCA classification. The JGCA N-category was defined by the anatomical extension of lymph node metastasis in association with the location of the primary tumor, while the TNM N-category was defined by number of metastatic regional lymph nodes. Items that are compatible in the JGCA classification and the TNM classification, and items that are not compatible are listed in Table 2 for convenience.

After the patients' data were entered with the data entry software, the patients' names and other personal information were removed from the exporting data set for privacy protection. A compact disk containing the linkable anonymous data was then mailed to the JGCA data center, located at Niigata University Medical and Dental Hospital. The accumulated data of the patients were reviewed and analyzed by the JGCA registration committee. One- to 5-year survival rates (5YSRs) were calculated for various subsets of prognostic factors by the Kaplan-Meier method. Deaths of any cause observed during 5 postoperative years were counted as events in the survival analysis. SPSS Ver. 15 software (SPSS, Chicago, IL, USA) was used for

Table 1 Registration data

\begin{tabular}{ll}
\hline Category & Item \\
\hline $\begin{array}{l}\text { Personal } \\
\text { information }\end{array}$ & Name of hospital, serial no., case no., ID no. ${ }^{\text {a }, \text { age, sex }}$ \\
$\begin{array}{l}\text { Follow-up } \\
\text { Surgery }\end{array}$ & $\begin{array}{l}\text { Date of follow-up, survival situation, causes of death } \\
\text { Date of operation, approach, operative procedure, LN dissection (D), organs resected together with stomach, type of } \\
\text { reconstruction }\end{array}$ \\
Pathology & $\begin{array}{l}\text { Anatomical subsite, macroscopic type, size of tumor, histological type, depth of tumor invasion, ly, v, number of dissected } \\
\text { LNs, number of metastatic LNs, N, PM/DM, CY }\end{array}$ \\
$\begin{array}{l}\text { JGCA final } \\
\text { diagnosis }\end{array}$ & $\begin{array}{l}\text { Depth of tumor invasion, adjacent structure involved, fN, H, P, M, curability, stage } \\
\text { UICC TNM } \\
\text { categories }\end{array}$ \\
\hline
\end{tabular}

$L N$ lymph node, $l y$ lymphatic invasion, $v$ venous invasion, $N$ extent of LN metastasis (JGCA), $P M / D M$ involvement of proximal and distal margin, $C Y$ peritoneal cytology, $f N$ extent of $\mathrm{LN}$ metastasis (final diagnosis), $H$ liver metastasis, $P$ peritoneal metastasis, $M$ distant metastasis, JGCA Japanese Gastric Cancer Association, UICC International Union Against Cancer

${ }^{\text {a }}$ ID no. was not exported to the registration data set 
Table 2 Compatibility to convert JGCA classification to TNM classification

\begin{tabular}{|c|c|c|c|}
\hline Category & JGCA 13th ed. & TNM 5th ed. & Compatibility \\
\hline $\mathrm{T}$ & $1-4$ & $0-4$ & Compatible \\
\hline \multirow[t]{2}{*}{$\mathrm{N}$} & 0 & 0 & Identical \\
\hline & $1-3$ & $1-3$ & Incompatible \\
\hline \multirow[t]{2}{*}{$\mathrm{M}^{\mathrm{a}}$} & 0 & 0 & Compatible \\
\hline & 1 & 1 & Compatible \\
\hline \multirow[t]{2}{*}{$\mathrm{H}$} & 0 & None & \\
\hline & 1 & M1 & Compatible \\
\hline \multirow[t]{2}{*}{$\mathrm{P}$} & 0 & None & \\
\hline & 1 & M1 & Compatible \\
\hline \multirow[t]{2}{*}{$\mathrm{CY}$} & 0 & None & \\
\hline & 1 & None & \\
\hline \multirow[t]{2}{*}{ Stage } & IA & IA & Identical \\
\hline & $\begin{array}{l}\text { IB, II, IIIA, IIIB, } \\
\text { IV }\end{array}$ & $\begin{array}{l}\text { IB, II, IIIA, } \\
\text { IIIB, IV }\end{array}$ & Incompatible \\
\hline \multirow{2}{*}{$\begin{array}{l}\text { Lymphatic } \\
\text { invasion }\end{array}$} & ly0 & L0 & Identical \\
\hline & ly1-3 & $\mathrm{L} 1$ & Compatible \\
\hline \multirow{3}{*}{$\begin{array}{l}\text { Venous } \\
\text { invasion }\end{array}$} & v0 & v0 & Identical \\
\hline & $\mathrm{v} 1-3$ & v1 & Compatible \\
\hline & None & v2 & \\
\hline \multirow[t]{2}{*}{$\begin{array}{l}\text { Histological } \\
\text { typing }\end{array}$} & $\begin{array}{l}\text { Differentiated } \\
\text { type }\end{array}$ & G1-2 & Compatible \\
\hline & $\begin{array}{l}\text { Undifferentiated } \\
\text { type }\end{array}$ & G3-4 & Compatible \\
\hline $\begin{array}{l}\text { Residual } \\
\text { tumor }\end{array}$ & Resection $\mathrm{A}-\mathrm{C}$ & R0-2 & Incompatible \\
\hline
\end{tabular}

a JGCA M-category is defined as distant metastases other than peritoneal, liver, or cytological metastases statistical analyses. This nationwide registration program was approved by the ethics committee of the JGCA.

\section{Results}

The data were collected from 187 participating hospitals across the country. The geographical distribution of the registered patients among Japan's 47 prefectures is illustrated in Fig. 1. More than 1000 patients per year were registered in the prefectures of Tokyo and Osaka; on the other hand, the number of registered patients was less than 100 in 15 prefectures. The hospital volumes in the participating hospitals are indicated in Fig. 2. The median hospital volume was 66 patients per year.

Data of 13067 patients who had undergone surgery in 2001 for primary gastric tumors were eventually accumulated. Of these, 88 patients with benign tumor or non-epithelial tumor were excluded from the analysis. Ninety-four patients who received endoscopic mucosal resection were also excluded. Data of 881 patients lacked essential items. Consequently, data of the remaining 12004 patients were used for the final analysis.

The results are shown in Tables 3, 4, 5, 6, 7, 8, 9, 10, 11, $12,13,14,15,16,17,18,19,20,21,22,23,24,25,26,27$, and 28; data in these Tables are for the total number of patients, survival rates by year, standard error of 5YSR, direct death within 30 postoperative days, numbers lost to follow-up within 5 years, 5-year survivors, and main causes of death (such as local and/or lymph node metastasis, peritoneal metastasis, liver metastasis, distant metastasis, recurrence at unknown site, other cancer and other
Fig. 1 Geographical distribution of the registered patients

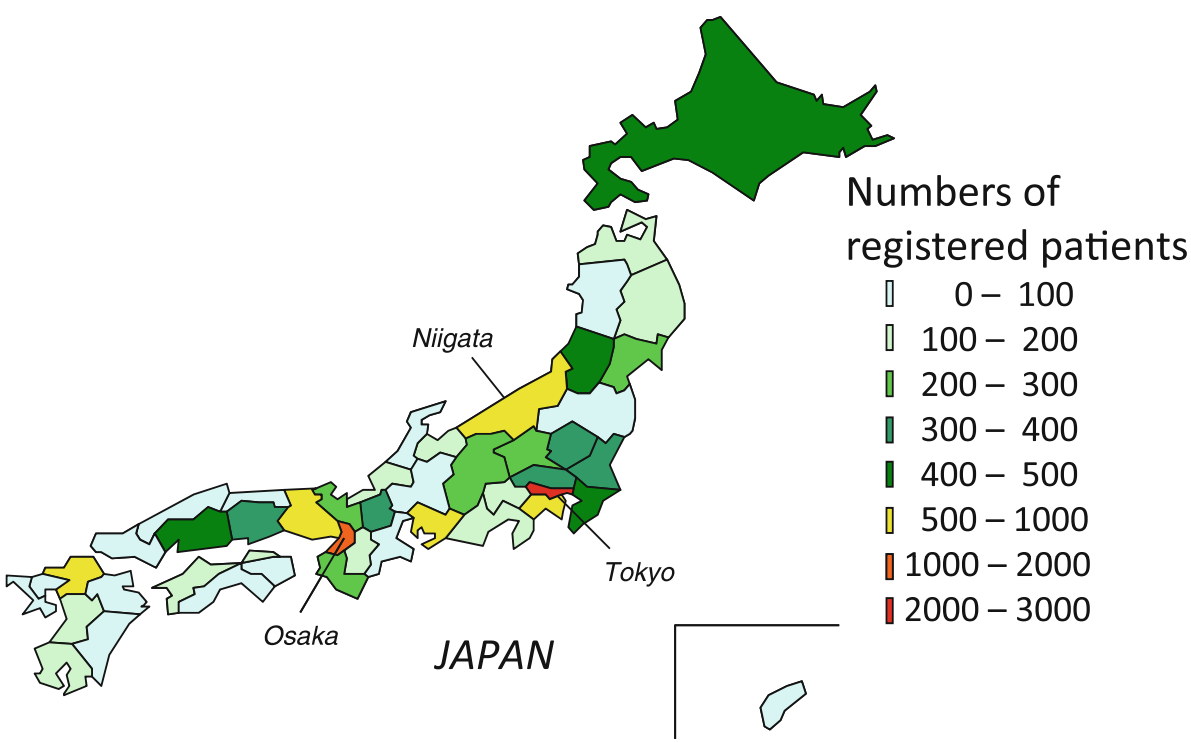




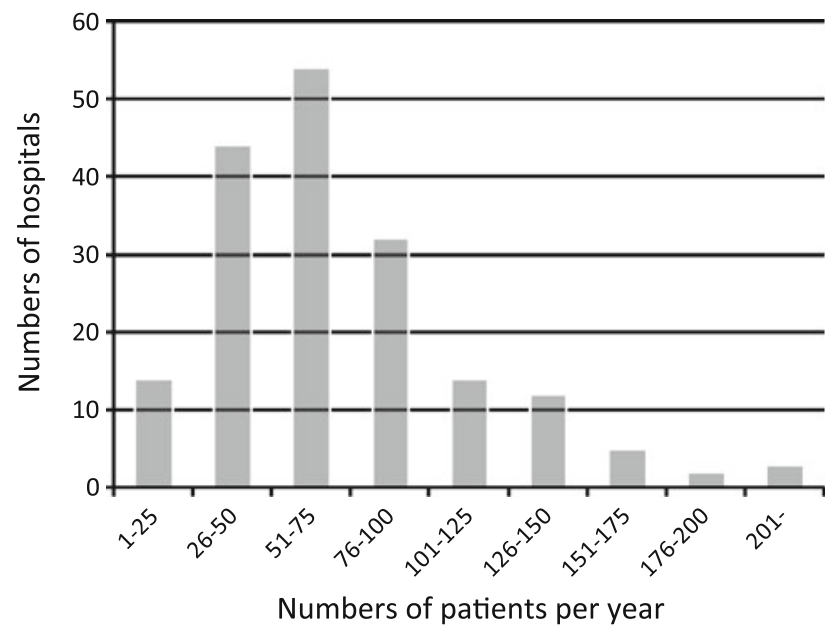

Fig. 2 Hospital volumes in the 187 participating hospitals disease). Figures 3, 4, 5, 6, 7, 8, 9, 10, 11, 12, 13, and 14 show cumulative survival curves of patients stratified by essential categories.

The 5YSR in the 12004 patients with primary gastric cancer was $69.1 \%$ (Table 3; Fig. 3). Within 5 postoperative years, 1976 patients were lost to follow-up; the follow-up rate was $83.5 \%$. Of the 12004 patients, 11261 underwent gastric resection; 350 were unresected; and in 393 the type of surgery was not specified. Accordingly, the resection rate was $97.0 \%$ (11261/11611). Sixty-three of the 11261 patients who had undergone gastrectomy died within 30 postoperative days; the direct death rate was $0.6 \%$ (Table 4; Fig. 4).

The most frequent cause of death in patients who had received gastrectomy was peritoneal metastasis $(n=$ 1040), followed, in descending order, by other diseases $(n=501)$, liver metastasis $(n=357)$, recurrence at an

Table 3 Survival outcomes of primary cancer

\begin{tabular}{|c|c|c|c|c|c|c|c|c|c|c|c|c|c|c|c|c|c|c|}
\hline & \multirow{2}{*}{$\begin{array}{l}\text { No. of } \\
\text { patients }\end{array}$} & \multicolumn{5}{|c|}{ Postoperative survival rate (\%) } & \multirow{2}{*}{$\begin{array}{l}\text { SE of } \\
5 Y S R\end{array}$} & \multirow[t]{2}{*}{ DD } & \multirow{2}{*}{$\begin{array}{l}\text { Lost to } \\
\text { follow up }\end{array}$} & \multirow[t]{2}{*}{ Alive } & \multicolumn{8}{|c|}{ Main cause of death } \\
\hline & & 1 year & 2 year & 3 year & 4 year & 5 year & & & & & $\mathrm{L}$ & $\mathrm{P}$ & $\mathrm{H}$ & M & $\mathrm{R}$ & $\mathrm{OC}$ & OD & UK \\
\hline $\begin{array}{r}\text { Primary } \\
\text { cancer }\end{array}$ & 12004 & 86.4 & 78.7 & 74.1 & 71.1 & 69.1 & 0.4 & 95 & 1976 & 6588 & 309 & 1266 & 374 & 183 & 349 & 162 & 530 & \\
\hline
\end{tabular}

$S E$ standard error, 5YSR 5-year survival rate, $D D$ direct death, Lost to follow up lost to follow-up within 5 years, Alive 5-year survivors, $L$ local recurrence and/or lymph node metastasis, $P$ peritoneal metastasis, $H$ liver metastasis, $M$ distant metastasis, $R$ recurrence at unknown site, $O C$ other cancer, $O D$ other disease, $U K$ unknown

Table 4 Survival outcomes of resected cases and unresected cases

\begin{tabular}{|c|c|c|c|c|c|c|c|c|c|c|c|c|c|c|c|c|c|c|}
\hline & \multirow{2}{*}{$\begin{array}{l}\text { No. of } \\
\text { patients }\end{array}$} & \multicolumn{5}{|c|}{ Postoperative survival rate (\%) } & \multirow{2}{*}{$\begin{array}{l}\text { SE of } \\
5 Y S R\end{array}$} & \multirow[t]{2}{*}{ DD } & \multirow{2}{*}{$\begin{array}{l}\text { Lost to } \\
\text { follow up }\end{array}$} & \multirow[t]{2}{*}{ Alive } & \multicolumn{8}{|c|}{ Main cause of death } \\
\hline & & 1 year & 2 year & 3 year & 4 year & 5 year & & & & & $\mathrm{L}$ & $\mathrm{P}$ & $\mathrm{H}$ & M & $\mathrm{R}$ & $\mathrm{OC}$ & OD & UK \\
\hline $\begin{array}{l}\text { Resected } \\
\text { cases }\end{array}$ & 11261 & 88.6 & 80.9 & 76.2 & 73.0 & 70.9 & 0.4 & 63 & 1877 & 6354 & 267 & 1040 & 357 & 161 & 298 & 155 & 501 & 251 \\
\hline $\begin{array}{l}\text { Unresected } \\
\text { cases }\end{array}$ & 350 & 23.0 & 9.8 & 7.1 & 5.6 & 5.3 & 1.3 & 20 & 40 & 14 & 32 & 176 & 12 & 13 & 43 & 0 & 10 & 10 \\
\hline
\end{tabular}

Table 5 Survival outcomes by sex

\begin{tabular}{|c|c|c|c|c|c|c|c|c|c|c|c|c|c|c|c|c|c|c|}
\hline & \multirow{2}{*}{$\begin{array}{l}\text { No. of } \\
\text { patients }\end{array}$} & \multicolumn{5}{|c|}{ Postoperative survival rate $(\%)$} & \multirow{2}{*}{$\begin{array}{l}\text { SE of } \\
5 Y S R\end{array}$} & \multirow[t]{2}{*}{ DD } & \multirow{2}{*}{$\begin{array}{l}\text { Lost to } \\
\text { follow } \\
\text { up }\end{array}$} & \multirow[t]{2}{*}{ Alive } & \multicolumn{8}{|c|}{ Main cause of death } \\
\hline & & 1 year & 2 year & 3 year & 4 year & 5 year & & & & & $\mathrm{L}$ & $\mathrm{P}$ & $\mathrm{H}$ & M & $\mathrm{R}$ & $\mathrm{OC}$ & OD & UK \\
\hline Male & 7828 & 88.4 & 80.7 & 75.6 & 72.3 & 70.0 & 0.5 & 47 & 1314 & 4348 & 190 & 646 & 299 & 112 & 205 & 138 & 403 & 173 \\
\hline Female & 3419 & 88.9 & 81.1 & 77.5 & 74.6 & 73.0 & 0.8 & 16 & 562 & 1997 & 76 & 392 & 58 & 49 & 93 & 17 & 97 & \\
\hline
\end{tabular}

Table 6 Survival outcomes by age

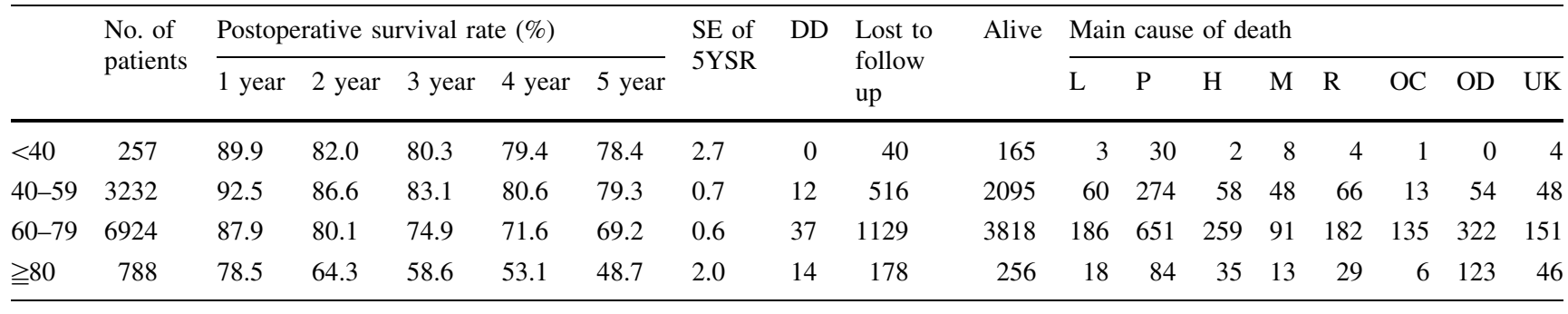


Table 7 Survival outcomes by tumor location

\begin{tabular}{|c|c|c|c|c|c|c|c|c|c|c|c|c|c|c|c|c|c|c|}
\hline & \multirow{2}{*}{$\begin{array}{l}\text { No. of } \\
\text { patients }\end{array}$} & \multicolumn{5}{|c|}{ Postoperative survival rate $(\%)$} & \multirow{2}{*}{$\begin{array}{l}\text { SE of } \\
5 Y S R\end{array}$} & \multirow[t]{2}{*}{ DD } & \multirow{2}{*}{$\begin{array}{l}\text { Lost to } \\
\text { follow up }\end{array}$} & \multirow[t]{2}{*}{ Alive } & \multicolumn{8}{|c|}{ Main cause of death } \\
\hline & & 1 year & 2 year & 3 year & 4 year & 5 year & & & & & $\mathrm{L}$ & $\mathrm{P}$ & $\mathrm{H}$ & M & $\mathrm{R}$ & $\mathrm{OC}$ & OD & UK \\
\hline $\mathrm{U}$ & 2399 & 86.0 & 76.7 & 71.3 & 67.5 & 65.3 & 1.0 & 13 & 370 & 1258 & 69 & 237 & 107 & 49 & 75 & 32 & 134 & 68 \\
\hline M & 4351 & 92.2 & 87.1 & 83.3 & 80.8 & 78.9 & 0.6 & 23 & 760 & 2741 & 65 & 260 & 90 & 43 & 84 & 65 & 161 & 82 \\
\hline $\mathrm{L}$ & 3936 & 89.4 & 81.4 & 77.1 & 74.2 & 71.9 & 0.7 & 21 & 685 & 2230 & 108 & 309 & 141 & 52 & 99 & 55 & 176 & 81 \\
\hline Whole & 532 & 63.7 & 44.7 & 33.7 & 25.8 & 23.4 & 2.0 & 6 & 56 & 104 & 23 & 230 & 17 & 17 & 34 & 3 & 28 & 20 \\
\hline
\end{tabular}

$U$ upper third, $M$ middle third, $L$ lower third of stomach

Table 8 Survival outcomes by macroscopic type

\begin{tabular}{|c|c|c|c|c|c|c|c|c|c|c|c|c|c|c|c|c|c|c|}
\hline & \multirow{2}{*}{$\begin{array}{l}\text { No. of } \\
\text { patients }\end{array}$} & \multicolumn{5}{|c|}{ Postoperative survival rate $(\%)$} & \multirow{2}{*}{$\begin{array}{l}\text { SE of } \\
5 Y S R\end{array}$} & \multirow[t]{2}{*}{ DD } & \multirow{2}{*}{$\begin{array}{l}\text { Lost to } \\
\text { follow up }\end{array}$} & \multirow[t]{2}{*}{ Alive } & \multicolumn{8}{|c|}{ Main cause of death } \\
\hline & & 1 year & 2 year & 3 year & 4 year & 5 year & & & & & $\mathrm{L}$ & $\mathrm{P}$ & $\mathrm{H}$ & M & $\mathrm{R}$ & $\mathrm{OC}$ & OD & UK \\
\hline Type 0 & 6085 & 97.5 & 95.7 & 93.7 & 91.8 & 90.3 & 0.4 & 12 & 1143 & 4401 & 20 & 45 & 23 & 23 & 32 & 100 & 217 & 81 \\
\hline Type 1 & 318 & 79.1 & 66.7 & 61.7 & 56.5 & 54.6 & 2.9 & 4 & 49 & 136 & 12 & 18 & 28 & 7 & 14 & 7 & 36 & 11 \\
\hline Type 2 & 1419 & 84.8 & 73.0 & 66.5 & 62.5 & 59.7 & 1.4 & 11 & 220 & 669 & 58 & 127 & 126 & 29 & 59 & 10 & 81 & 40 \\
\hline Type 3 & 2151 & 76.5 & 60.8 & 52.4 & 47.8 & 45.1 & 1.1 & 21 & 306 & 760 & 119 & 425 & 152 & 62 & 124 & 25 & 112 & 66 \\
\hline Type 4 & 779 & 62.1 & 41.9 & 30.0 & 23.4 & 20.4 & 1.5 & 10 & 65 & 133 & 37 & 363 & 11 & 31 & 54 & 7 & 35 & 43 \\
\hline Type 5 & 340 & 86.8 & 74.3 & 67.4 & 62.6 & 59.5 & 2.8 & 4 & 48 & 166 & 13 & 49 & 16 & 7 & 15 & 4 & 15 & 7 \\
\hline
\end{tabular}

Table 9 Survival outcomes by histological diagnosis

\begin{tabular}{|c|c|c|c|c|c|c|c|c|c|c|c|c|c|c|c|c|c|c|}
\hline & \multirow{2}{*}{$\begin{array}{l}\text { No. of } \\
\text { patients }\end{array}$} & \multicolumn{5}{|c|}{ Postoperative survival rate $(\%)$} & \multirow{2}{*}{$\begin{array}{l}\text { SE of } \\
5 Y S R\end{array}$} & \multirow[t]{2}{*}{ DD } & \multirow{2}{*}{$\begin{array}{l}\text { Lost to } \\
\text { follow up }\end{array}$} & \multirow[t]{2}{*}{ Alive } & \multicolumn{8}{|c|}{ Main cause of death } \\
\hline & & 1 year & 2 year & 3 year & 4 year & 5 year & & & & & $\mathrm{L}$ & $\mathrm{P}$ & $\mathrm{H}$ & M & $\mathrm{R}$ & $\mathrm{OC}$ & OD & UK \\
\hline pap & 364 & 85.8 & 75.1 & 70.4 & 67.5 & 65.1 & 2.6 & 3 & 64 & 185 & 11 & 27 & 23 & 6 & 13 & 8 & 23 & 4 \\
\hline tub1 & 2752 & 95.2 & 91.1 & 87.9 & 85.3 & 83.5 & 0.7 & 5 & 519 & 1818 & 30 & 55 & 42 & 16 & 36 & 51 & 137 & 48 \\
\hline tub2 & 2997 & 89.2 & 81.4 & 76.3 & 73.1 & 70.6 & 0.9 & 20 & 537 & 1651 & 64 & 207 & 156 & 46 & 74 & 45 & 160 & 57 \\
\hline por1 & 1476 & 82.5 & 72.4 & 67.8 & 64.9 & 63.7 & 1.3 & 14 & 238 & 737 & 53 & 174 & 82 & 30 & 40 & 14 & 69 & 39 \\
\hline por2 & 1903 & 81.4 & 69.7 & 63.4 & 59.5 & 56.6 & 1.2 & 15 & 244 & 886 & 75 & 401 & 34 & 44 & 86 & 19 & 59 & 55 \\
\hline sig & 1325 & 93.2 & 88.0 & 84.5 & 81.2 & 79.4 & 1.2 & 4 & 217 & 855 & 17 & 108 & 2 & 14 & 32 & 12 & 30 & 38 \\
\hline muc & 231 & 81.5 & 68.8 & 60.4 & 53.7 & 51.2 & 3.4 & 1 & 24 & 100 & 9 & 54 & 5 & 1 & 10 & 3 & 19 & 6 \\
\hline $\begin{array}{l}\text { Adenosquamous } \\
\text { carcinoma }\end{array}$ & 6 & 50.0 & 33.3 & 33.3 & 16.7 & 16.7 & 15.2 & 0 & 0 & 1 & 0 & 2 & 2 & 0 & 0 & 1 & 0 & 0 \\
\hline $\begin{array}{l}\text { Squamous cell } \\
\text { carcinoma }\end{array}$ & 5 & 60.0 & 30.0 & 0.0 & 0.0 & 0.0 & 0.0 & 0 & 1 & 0 & 2 & 1 & 0 & 1 & 0 & 0 & 0 & 0 \\
\hline $\begin{array}{l}\text { Miscellaneous } \\
\text { carcinoma }\end{array}$ & 45 & 65.2 & 53.1 & 48.1 & 45.6 & 45.6 & 7.7 & 0 & 4 & 18 & 2 & 8 & 7 & 2 & 2 & 0 & 1 & 1 \\
\hline
\end{tabular}

Pap papillary adenocarcinoma, tubl tubular adenocarcinoma, well-differentiated type, tub2 tubular adenocarcinoma, moderately differentiated type, porl poorly differentiated adenocarcinoma, solid type, por2 poorly differentiated adenocarcinoma, non-solid type, sig signet-ring cell carcinoma, mис mucinous adenocarcinoma

Table 10 Survival outcomes by histological differentiation

\begin{tabular}{|c|c|c|c|c|c|c|c|c|c|c|c|c|c|c|c|c|c|c|}
\hline & \multirow{2}{*}{$\begin{array}{l}\text { No. of } \\
\text { patients }\end{array}$} & \multicolumn{5}{|c|}{ Postoperative survival rate (\%) } & \multirow{2}{*}{$\begin{array}{l}\text { SE of } \\
5 Y S R\end{array}$} & \multirow[t]{2}{*}{ DD } & \multirow{2}{*}{$\begin{array}{l}\text { Lost to } \\
\text { follow } \\
\text { up }\end{array}$} & \multirow[t]{2}{*}{ Alive } & \multicolumn{8}{|c|}{ Main cause of death } \\
\hline & & 1 year & 2 year & 3 year & 4 year & 5 year & & & & & $\mathrm{L}$ & $\mathrm{P}$ & $\mathrm{H}$ & M & $\mathrm{R}$ & $\mathrm{OC}$ & OD & UK \\
\hline $\begin{array}{l}\text { Differentiated } \\
\text { type }\end{array}$ & 6113 & 91.7 & 85.4 & 81.2 & 78.3 & 76.1 & 0.6 & 28 & 1120 & 3654 & 105 & 289 & 221 & 68 & 123 & 104 & 320 & 109 \\
\hline $\begin{array}{l}\text { Undifferentiated } \\
\text { type }\end{array}$ & 4935 & 84.9 & 75.4 & 70.1 & 66.6 & 64.6 & 0.7 & 34 & 723 & 2578 & 154 & 737 & 123 & 89 & 168 & 48 & 177 & 138 \\
\hline Other type & 144 & 81.6 & 75.3 & 71.9 & 68.4 & 68.4 & 4.1 & 1 & 29 & 74 & 6 & 12 & 11 & 4 & 2 & 1 & 3 & \\
\hline
\end{tabular}


Table 11 Survival outcomes by venous invasion (v)

\begin{tabular}{|c|c|c|c|c|c|c|c|c|c|c|c|c|c|c|c|c|c|c|}
\hline & \multirow{2}{*}{$\begin{array}{l}\text { No. of } \\
\text { patients }\end{array}$} & \multicolumn{5}{|c|}{ Postoperative survival rate $(\%)$} & \multirow{2}{*}{$\begin{array}{l}\text { SE of } \\
5 Y S R\end{array}$} & \multirow[t]{2}{*}{ DD } & \multirow{2}{*}{$\begin{array}{l}\text { Lost to } \\
\text { follow up }\end{array}$} & \multirow[t]{2}{*}{ Alive } & \multicolumn{8}{|c|}{ Main cause of death } \\
\hline & & 1 year & 2 year & 3 year & 4 year & 5 year & & & & & $\mathrm{L}$ & $P$ & $\mathrm{H}$ & M & $\mathrm{R}$ & $\mathrm{OC}$ & OD & UK \\
\hline v0 & 6453 & 95.4 & 91.5 & 88.6 & 86.2 & 84.5 & 0.5 & 23 & 1228 & 4304 & 54 & 258 & 59 & 36 & 70 & 101 & 260 & 83 \\
\hline v1 & 2601 & 84.5 & 72.7 & 66.6 & 62.2 & 59.7 & 1.0 & 17 & 352 & 1276 & 103 & 365 & 115 & 53 & 112 & 29 & 127 & 69 \\
\hline v2 & 1347 & 75.7 & 59.8 & 50.4 & 45.8 & 42.6 & 1.4 & 17 & 168 & 463 & 71 & 271 & 95 & 44 & 74 & 16 & 84 & 61 \\
\hline v3 & 539 & 59.4 & 44.5 & 35.7 & 32.2 & 30.8 & 2.1 & 5 & 69 & 128 & 30 & 123 & 85 & 23 & 34 & 4 & 21 & 22 \\
\hline
\end{tabular}

Table 12 Survival outcomes by lymphatic invasion (ly)

\begin{tabular}{|c|c|c|c|c|c|c|c|c|c|c|c|c|c|c|c|c|c|c|}
\hline & \multirow{2}{*}{$\begin{array}{l}\text { No. of } \\
\text { patients }\end{array}$} & \multicolumn{5}{|c|}{ Postoperative survival rate (\%) } & \multirow{2}{*}{$\begin{array}{l}\text { SE of } \\
5 Y S R\end{array}$} & \multirow[t]{2}{*}{ DD } & \multirow{2}{*}{$\begin{array}{l}\text { Lost to } \\
\text { follow up }\end{array}$} & \multirow[t]{2}{*}{ Alive } & \multicolumn{8}{|c|}{ Main cause of death } \\
\hline & & 1 year & 2 year & 3 year & 4 year & 5 year & & & & & $\mathrm{L}$ & $\mathrm{P}$ & $\mathrm{H}$ & M & $\mathrm{R}$ & $\mathrm{OC}$ & OD & UK \\
\hline ly0 & 4783 & 97.2 & 95.3 & 93.3 & 91.4 & 89.9 & 0.5 & 11 & 956 & 3389 & 10 & 48 & 23 & 11 & 35 & 80 & 177 & 54 \\
\hline ly1 & 2604 & 92.4 & 86.1 & 81.1 & 77.7 & 75.1 & 0.9 & 13 & 398 & 1606 & 51 & 187 & 84 & 36 & 37 & 40 & 115 & 50 \\
\hline ly 2 & 2047 & 80.7 & 65.8 & 58.4 & 53.3 & 50.5 & 1.2 & 22 & 271 & 834 & 102 & 346 & 134 & 53 & 103 & 17 & 123 & 64 \\
\hline ly3 & 1481 & 65.2 & 45.4 & 36.3 & 31.6 & 29.4 & 1.3 & 16 & 194 & 334 & 95 & 438 & 110 & 57 & 110 & 13 & 77 & 53 \\
\hline
\end{tabular}

Table 13 Survival outcomes by depth of invasion

\begin{tabular}{|c|c|c|c|c|c|c|c|c|c|c|c|c|c|c|c|c|c|c|}
\hline & \multirow{2}{*}{$\begin{array}{l}\text { No. of } \\
\text { patients }\end{array}$} & \multicolumn{5}{|c|}{ Postoperative survival rate $(\%)$} & \multirow{2}{*}{$\begin{array}{l}\text { SE of } \\
5 Y S R\end{array}$} & \multirow[t]{2}{*}{ DD } & \multirow{2}{*}{$\begin{array}{l}\text { Lost to } \\
\text { follow } \\
\text { up }\end{array}$} & \multirow[t]{2}{*}{ Alive } & \multicolumn{8}{|c|}{ Main cause of death } \\
\hline & & 1 year & 2 year & 3 year & 4 year & 5 year & & & & & $\mathrm{L}$ & $\mathrm{P}$ & $\mathrm{H}$ & M & $\mathrm{R}$ & $\mathrm{OC}$ & OD & UK \\
\hline pT1(M) & 3071 & 98.1 & 96.9 & 95.0 & 93.5 & 92.2 & 0.5 & 5 & 606 & 2248 & 7 & 4 & 4 & 1 & 7 & 53 & 98 & 43 \\
\hline $\mathrm{pT} 1(\mathrm{SM})$ & 2662 & 97.5 & 95.0 & 93.1 & 90.9 & 89.1 & 0.6 & 6 & 500 & 1898 & 11 & 16 & 19 & 11 & 16 & 51 & 109 & 31 \\
\hline pT2(MP) & 1071 & 93.4 & 88.7 & 84.0 & 80.9 & 78.3 & 1.3 & 3 & 183 & 675 & 13 & 23 & 31 & 19 & 22 & 17 & 68 & 20 \\
\hline pT2(SS) & 1695 & 87.0 & 74.7 & 67.6 & 63.2 & 60.6 & 1.2 & 17 & 262 & 817 & 67 & 148 & 122 & 48 & 65 & 20 & 99 & 47 \\
\hline pT3(SE) & 2278 & 69.7 & 50.9 & 41.3 & 35.8 & 33.0 & 1.0 & 26 & 264 & 601 & 132 & 712 & 140 & 72 & 148 & 10 & 102 & 97 \\
\hline pT4(SI) & 417 & 57.7 & 38.1 & 30.0 & 26.0 & 22.8 & 2.2 & 5 & 45 & 77 & 36 & 134 & 39 & 8 & 40 & 4 & 24 & 10 \\
\hline
\end{tabular}

$p$ pathological finding, $M$ mucosa or muscuralis musoca, $S M$ submucosa, $M P$ muscularis propria, $S S$ subserosal, $S E$ serosa, $S I$ adjacent structures

Table 14 Survival outcomes by p classification

\begin{tabular}{|c|c|c|c|c|c|c|c|c|c|c|c|c|c|c|c|c|c|c|}
\hline & \multirow{2}{*}{$\begin{array}{l}\text { No. of } \\
\text { patients }\end{array}$} & \multicolumn{5}{|c|}{ Postoperative survival rate $(\%)$} & \multirow{2}{*}{$\begin{array}{l}\text { SE of } \\
5 Y S R\end{array}$} & \multirow[t]{2}{*}{ DD } & \multirow{2}{*}{$\begin{array}{l}\text { Lost to } \\
\text { follow up }\end{array}$} & \multirow[t]{2}{*}{ Alive } & \multicolumn{8}{|c|}{ Main cause of death } \\
\hline & & 1 year & 2 year & 3 year & 4 year & 5 year & & & & & $\mathrm{L}$ & $\mathrm{P}$ & $\mathrm{H}$ & M & $\mathrm{R}$ & $\mathrm{OC}$ & OD & UK \\
\hline pT1 & 5733 & 97.8 & 96.0 & 94.1 & 92.3 & 90.8 & 0.4 & 11 & 1106 & 4146 & 18 & 20 & 23 & 12 & 23 & 104 & 207 & 74 \\
\hline pT2 & 2766 & 89.5 & 80.1 & 74.0 & 70.1 & 67.5 & 0.9 & 20 & 445 & 1492 & 80 & 171 & 153 & 67 & 87 & 37 & 167 & 67 \\
\hline pT3 & 2278 & 69.7 & 50.9 & 41.3 & 35.8 & 33.0 & 1.0 & 26 & 264 & 601 & 132 & 712 & 140 & 72 & 148 & 10 & 102 & 97 \\
\hline pT4 & 417 & 57.7 & 38.1 & 30.0 & 26.0 & 22.8 & 2.2 & 5 & 45 & 77 & 36 & 134 & 39 & 8 & 40 & 4 & 24 & 10 \\
\hline
\end{tabular}

Table 15 Survival outcomes by lymph node metastasis $(\mathrm{pN})$

\begin{tabular}{|c|c|c|c|c|c|c|c|c|c|c|c|c|c|c|c|c|c|c|}
\hline & \multirow{2}{*}{$\begin{array}{l}\text { No. of } \\
\text { patients }\end{array}$} & \multicolumn{5}{|c|}{ Postoperative survival rate $(\%)$} & \multirow{2}{*}{$\begin{array}{l}\text { SE of } \\
5 Y S R\end{array}$} & \multirow[t]{2}{*}{ DD } & \multirow{2}{*}{$\begin{array}{l}\text { Lost to } \\
\text { follow up }\end{array}$} & \multirow[t]{2}{*}{ Alive } & \multicolumn{8}{|c|}{ Main cause of death } \\
\hline & & 1 year & 2 year & 3 year & 4 year & 5 year & & & & & $\mathrm{L}$ & $\mathrm{P}$ & $\mathrm{H}$ & M & $\mathrm{R}$ & $\mathrm{OC}$ & OD & UK \\
\hline pN0 & 6508 & 97.0 & 94.7 & 92.5 & 90.6 & 89.0 & 0.4 & 22 & 1240 & 4616 & 18 & 95 & 38 & 16 & 44 & 109 & 248 & 84 \\
\hline pN1 & 2274 & 84.7 & 72.3 & 66.2 & 61.3 & 58.3 & 1.1 & 12 & 322 & 1074 & 78 & 309 & 139 & 46 & 99 & 23 & 118 & 66 \\
\hline pN2 & 1703 & 72.1 & 52.8 & 41.4 & 35.8 & 33.4 & 1.2 & 19 & 224 & 439 & 103 & 442 & 135 & 69 & 109 & 13 & 100 & 69 \\
\hline pN3 & 421 & 53.8 & 33.1 & 25.8 & 22.0 & 17.4 & 1.9 & 4 & 33 & 61 & 60 & 136 & 37 & 28 & 35 & 3 & 13 & 15 \\
\hline
\end{tabular}


Table 16 Survival outcomes by liver metastasis (fH)

\begin{tabular}{|c|c|c|c|c|c|c|c|c|c|c|c|c|c|c|c|c|c|c|}
\hline & \multirow{2}{*}{$\begin{array}{l}\text { No. of } \\
\text { patients }\end{array}$} & \multicolumn{5}{|c|}{ Postoperative survival rate $(\%)$} & \multirow{2}{*}{$\begin{array}{l}\text { SE of } \\
5 Y S R\end{array}$} & \multirow[t]{2}{*}{ DD } & \multirow{2}{*}{$\begin{array}{l}\text { Lost to } \\
\text { follow up }\end{array}$} & \multirow[t]{2}{*}{ Alive } & \multicolumn{8}{|c|}{ Main cause of death } \\
\hline & & 1 year & 2 year & 3 year & 4 year & 5 year & & & & & $\mathrm{L}$ & $\mathrm{P}$ & $\mathrm{H}$ & M & $\mathrm{R}$ & $\mathrm{OC}$ & OD & UK \\
\hline fHO & 10665 & 89.9 & 82.6 & 78.1 & 74.9 & 72.7 & 0.5 & 55 & 1806 & 6171 & 249 & 956 & 216 & 143 & 268 & 144 & 482 & 230 \\
\hline fH1 & 305 & 42.6 & 24.6 & 15.3 & 12.2 & 11.8 & 2.0 & 7 & 28 & 28 & 8 & 48 & 130 & 15 & 25 & 5 & 10 & 8 \\
\hline
\end{tabular}

$f$ final finding

Table 17 Survival outcomes by peritoneal metastasis (fP)

\begin{tabular}{|c|c|c|c|c|c|c|c|c|c|c|c|c|c|c|c|c|c|c|}
\hline & \multirow{2}{*}{$\begin{array}{l}\text { No. of } \\
\text { patients }\end{array}$} & \multicolumn{5}{|c|}{ Postoperative survival rate $(\%)$} & \multirow{2}{*}{$\begin{array}{l}\text { SE of } \\
5 Y S R\end{array}$} & \multirow[t]{2}{*}{ DD } & \multirow{2}{*}{$\begin{array}{l}\text { Lost to } \\
\text { follow up }\end{array}$} & \multirow[t]{2}{*}{ Alive } & \multicolumn{8}{|c|}{ Main cause of death } \\
\hline & & 1 year & 2 year & 3 year & 4 year & 5 year & & & & & $\mathrm{L}$ & $\mathrm{P}$ & $\mathrm{H}$ & M & $\mathrm{R}$ & $\mathrm{OC}$ & OD & UK \\
\hline $\mathrm{fP0}$ & 10301 & 91.2 & 84.5 & 80.0 & 76.9 & 74.8 & 0.4 & 49 & 1771 & 6131 & 232 & 628 & 322 & 143 & 245 & 148 & 468 & 213 \\
\hline fP1 & 658 & 49.0 & 27.0 & 19.3 & 14.7 & 12.4 & 1.4 & 11 & 64 & 66 & 24 & 363 & 30 & 15 & 49 & 1 & 21 & 25 \\
\hline
\end{tabular}

Table 18 Survival outcomes by peritoneal cytology (CY)

\begin{tabular}{|c|c|c|c|c|c|c|c|c|c|c|c|c|c|c|c|c|c|c|}
\hline & \multirow{2}{*}{$\begin{array}{l}\text { No. of } \\
\text { patients }\end{array}$} & \multicolumn{5}{|c|}{ Postoperative survival rate $(\%)$} & \multirow{2}{*}{$\begin{array}{l}\text { SE of } \\
5 Y S R\end{array}$} & \multirow[t]{2}{*}{ DD } & \multirow{2}{*}{$\begin{array}{l}\text { Lost to } \\
\text { follow up }\end{array}$} & \multirow[t]{2}{*}{ Alive } & \multicolumn{8}{|c|}{ Main cause of death } \\
\hline & & 1 year & 2 year & 3 year & 4 year & 5 year & & & & & $\mathrm{L}$ & $\mathrm{P}$ & $\mathrm{H}$ & M & $\mathrm{R}$ & $\mathrm{OC}$ & OD & UK \\
\hline CY0 & 4109 & 88.6 & 78.9 & 73.0 & 68.9 & 66.4 & 0.8 & 24 & 671 & 2157 & 135 & 403 & 184 & 82 & 120 & 56 & 185 & 116 \\
\hline CY1 & 651 & 51.6 & 29.1 & 18.2 & 14.9 & 12.3 & 1.4 & 4 & 73 & 60 & 23 & 338 & 35 & 15 & 62 & 4 & 25 & 16 \\
\hline
\end{tabular}

Table 19 Survival outcomes by distant metastasis (fM)

\begin{tabular}{|c|c|c|c|c|c|c|c|c|c|c|c|c|c|c|c|c|c|c|}
\hline & \multirow{2}{*}{$\begin{array}{l}\text { No. of } \\
\text { patients }\end{array}$} & \multicolumn{5}{|c|}{ Postoperative survival rate $(\%)$} & \multirow{2}{*}{$\begin{array}{l}\text { SE of } \\
5 Y S R\end{array}$} & \multirow[t]{2}{*}{ DD } & \multirow{2}{*}{$\begin{array}{l}\text { Lost to } \\
\text { follow up }\end{array}$} & \multirow[t]{2}{*}{ Alive } & \multicolumn{8}{|c|}{ Main cause of death } \\
\hline & & 1 year & 2 year & 3 year & 4 year & 5 year & & & & & $\mathrm{L}$ & $\mathrm{P}$ & $\mathrm{H}$ & M & $\mathrm{R}$ & $\mathrm{OC}$ & OD & UK \\
\hline fM0 & 10752 & 89.4 & 82.0 & 77.3 & 74.2 & 72.1 & 0.5 & 59 & 1817 & 6159 & 233 & 932 & 331 & 140 & 278 & 149 & 479 & 234 \\
\hline fM1 & 215 & 46.7 & 27.3 & 23.6 & 19.7 & 18.0 & 2.8 & 3 & 21 & 30 & 25 & 72 & 15 & 16 & 16 & 2 & 14 & 4 \\
\hline
\end{tabular}

Table 20 Survival outcomes by JGCA stage

\begin{tabular}{|c|c|c|c|c|c|c|c|c|c|c|c|c|c|c|c|c|c|c|}
\hline & \multirow{2}{*}{$\begin{array}{l}\text { No. of } \\
\text { patients }\end{array}$} & \multicolumn{5}{|c|}{ Postoperative survival rate (\%) } & \multirow{2}{*}{$\begin{array}{l}\text { SE of } \\
5 Y S R\end{array}$} & \multirow[t]{2}{*}{$\mathrm{DD}$} & \multirow{2}{*}{$\begin{array}{l}\text { Lost to } \\
\text { follow up }\end{array}$} & \multirow[t]{2}{*}{ Alive } & \multicolumn{8}{|c|}{ Main cause of death } \\
\hline & & 1 year & 2 year & 3 year & 4 year & 5 year & & & & & $\mathrm{L}$ & $\mathrm{P}$ & $\mathrm{H}$ & M & $\mathrm{R}$ & $\mathrm{OC}$ & OD & UK \\
\hline Stage IA & 4997 & 98.2 & 96.7 & 94.9 & 93.2 & 91.9 & 0.4 & 11 & 983 & 3646 & 6 & 11 & 8 & 3 & 14 & 87 & 181 & 58 \\
\hline Stage IB & 1459 & 96.4 & 93.0 & 90.1 & 87.4 & 85.1 & 1.0 & 7 & 267 & 993 & 9 & 28 & 13 & 11 & 15 & 28 & 78 & 17 \\
\hline Stage II & 1237 & 93.0 & 85.0 & 79.7 & 75.7 & 73.1 & 1.3 & 7 & 196 & 736 & 26 & 70 & 44 & 24 & 38 & 14 & 65 & 24 \\
\hline Stage IIIA & 975 & 85.8 & 71.2 & 61.2 & 55.2 & 51.0 & 1.7 & 9 & 143 & 395 & 47 & 137 & 50 & 32 & 53 & 6 & 61 & 51 \\
\hline Stage IIIB & 562 & 76.6 & 55.3 & 43.9 & 36.0 & 33.4 & 2.1 & 5 & 63 & 153 & 48 & 141 & 31 & 24 & 40 & 2 & 36 & 24 \\
\hline Stage IV & 1649 & 53.9 & 32.2 & 22.4 & 18.3 & 15.8 & 1.0 & 22 & 161 & 206 & 122 & 626 & 199 & 62 & 135 & 11 & 71 & 56 \\
\hline
\end{tabular}

unknown site $(n=298)$, and local recurrence including node metastasis $(n=267)$.

The proportion of male patients was $69.6 \%$ and their 5YSR was lower than that of female patients $(P<0.01$;

Table 5; Fig. 5). The proportion of patients who were more than 80 years old was $7.0 \%$, and their 5YSR was $48.7 \%$ (Table 6; Fig. 6). Upper-third gastric cancer accounted for $21.4 \%$ of the cases, and the 5YSR $(65.3 \%)$ of patients with cancer at this site was lower than that for the middle- and lower-third cancers $(P<0.001 ;$ Table 7; Fig. 7). The 
Table 21 Survival outcomes by JGCA stage (4 classifications)

\begin{tabular}{|c|c|c|c|c|c|c|c|c|c|c|c|c|c|c|c|c|c|c|}
\hline & \multirow{2}{*}{$\begin{array}{l}\text { No. of } \\
\text { patients }\end{array}$} & \multicolumn{5}{|c|}{ Postoperative survival rate $(\%)$} & \multirow{2}{*}{$\begin{array}{l}\text { SE of } \\
5 Y S R\end{array}$} & \multirow[t]{2}{*}{ DD } & \multirow{2}{*}{$\begin{array}{l}\text { Lost to } \\
\text { follow up }\end{array}$} & \multirow[t]{2}{*}{ Alive } & \multicolumn{8}{|c|}{ Main cause of death } \\
\hline & & 1 year & 2 year & 3 year & 4 year & 5 year & & & & & $\mathrm{L}$ & $\mathrm{P}$ & $\mathrm{H}$ & M & $\mathrm{R}$ & OC & OD & UK \\
\hline Stage I & 6456 & 97.8 & 95.8 & 93.8 & 91.9 & 90.3 & 0.4 & 18 & 1250 & 4639 & 15 & 39 & 21 & 14 & 29 & 115 & 259 & 75 \\
\hline Stage II & 1237 & 93.0 & 85.0 & 79.7 & 75.7 & 73.1 & 1.3 & 7 & 196 & 736 & 26 & 70 & 44 & 24 & 38 & 14 & 65 & 24 \\
\hline Stage III & 1537 & 82.4 & 65.4 & 54.9 & 48.2 & 44.5 & 1.3 & 14 & 206 & 548 & 95 & 278 & 81 & 56 & 93 & 8 & 97 & 75 \\
\hline Stage IV & 1649 & 53.9 & 32.2 & 22.4 & 18.3 & 15.8 & 1.0 & 22 & 161 & 206 & 122 & 626 & 199 & 62 & 135 & 11 & 71 & 56 \\
\hline
\end{tabular}

Table 22 Survival outcomes by TNM stage

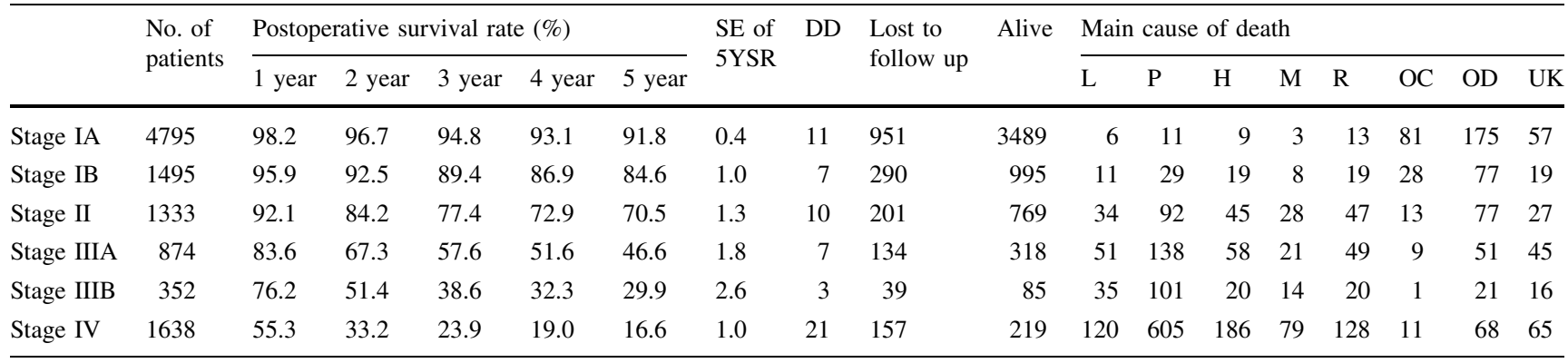

Table 23 Survival outcomes by TNM stage (4 classifications)

\begin{tabular}{|c|c|c|c|c|c|c|c|c|c|c|c|c|c|c|c|c|c|c|}
\hline & \multirow{2}{*}{$\begin{array}{l}\text { No. of } \\
\text { patients }\end{array}$} & \multicolumn{5}{|c|}{ Postoperative survival rate $(\%)$} & \multirow{2}{*}{$\begin{array}{l}\text { SE of } \\
5 Y S R\end{array}$} & \multirow[t]{2}{*}{ DD } & \multirow{2}{*}{$\begin{array}{l}\text { Lost to } \\
\text { follow } \\
\text { up }\end{array}$} & \multirow[t]{2}{*}{ Alive } & \multicolumn{8}{|c|}{ Main cause of death } \\
\hline & & 1 year & 2 year & 3 year & 4 year & 5 year & & & & & $\mathrm{L}$ & $\mathrm{P}$ & $\mathrm{H}$ & M & $\mathrm{R}$ & $\mathrm{OC}$ & OD & UK \\
\hline Stage I & 6290 & 97.7 & 95.7 & 93.5 & 91.7 & 90.1 & 0.4 & 18 & 1241 & 4484 & 17 & 40 & 28 & 11 & 32 & 109 & 252 & 76 \\
\hline Stage II & 1333 & 92.1 & 84.2 & 77.4 & 72.9 & 70.5 & 1.3 & 10 & 201 & 769 & 34 & 92 & 45 & 28 & 47 & 13 & 77 & 27 \\
\hline Stage III & 1226 & 81.4 & 62.7 & 52.1 & 46.0 & 41.8 & 1.5 & 10 & 173 & 403 & 86 & 239 & 78 & 35 & 69 & 10 & 72 & 61 \\
\hline Stage IV & 1638 & 55.3 & 33.2 & 23.9 & 19.0 & 16.6 & 1.0 & 21 & 157 & 219 & 120 & 605 & 186 & 79 & 128 & 11 & 68 & 65 \\
\hline
\end{tabular}

Table 24 Survival outcomes by approaches

\begin{tabular}{|c|c|c|c|c|c|c|c|c|c|c|c|c|c|c|c|c|c|c|}
\hline & \multirow{2}{*}{$\begin{array}{l}\text { No. of } \\
\text { patients }\end{array}$} & \multicolumn{5}{|c|}{ Postoperative survival rate (\%) } & \multirow{2}{*}{$\begin{array}{l}\text { SE of } \\
5 Y S R\end{array}$} & \multirow[t]{2}{*}{ DD } & \multirow{2}{*}{$\begin{array}{l}\text { Lost to } \\
\text { follow up }\end{array}$} & \multirow[t]{2}{*}{ Alive } & \multicolumn{8}{|c|}{ Main cause of death } \\
\hline & & 1 year & 2 year & 3 year & 4 year & 5 year & & & & & $\mathrm{L}$ & $\mathrm{P}$ & $\mathrm{H}$ & $\mathrm{M}$ & $\mathrm{R}$ & $\mathrm{OC}$ & OD & UK \\
\hline Laparotomy & 10532 & 88.3 & 80.4 & 75.6 & 72.4 & 70.2 & 0.5 & 59 & 1757 & 5869 & 251 & 1002 & 345 & 154 & 289 & 147 & 487 & 231 \\
\hline $\begin{array}{l}\text { Thoraco- } \\
\text { laparotomy }\end{array}$ & 112 & 70.5 & 56.0 & 47.6 & 43.7 & 40.7 & 4.7 & 3 & 8 & 39 & 14 & 19 & 11 & 6 & 7 & 0 & 4 & 4 \\
\hline Laparoscopic & 396 & 99.2 & 98.9 & 98.6 & 97.7 & 97.4 & 0.9 & 0 & 87 & 300 & 0 & 0 & 0 & 0 & 1 & 2 & 3 & 3 \\
\hline Others & 2 & 100.0 & 50.0 & 50.0 & 50.0 & 50.0 & 35.4 & 0 & 0 & 1 & 0 & 0 & 0 & 0 & 0 & 0 & 1 & 0 \\
\hline
\end{tabular}

proportion of patients with type 4 cancer was $7.0 \%$, and their 5YSR was markedly low, at 20.4\% $(P<0.001$; Table 8; Fig. 8). In regard to the histological type, the $5 Y S R$ of patients with undifferentiated type, including poorly differentiated adenocarcinoma, signet-ring cell carcinoma, and mucinous adenocarcinoma, was $64.6 \%$. The undifferentiated type showed a poorer prognosis than the differentiated type $(P<0.001$; Tables 9,10$)$. The grade of venous invasion $(\mathrm{v} 0-\mathrm{v} 3)$ and that of lymphatic invasion (ly0-1y3) showed significant correlations with prognosis $(P<0.001$; Tables 11, 12).

There was a high incidence of early-stage cancer, as indicated in Tables 13 and 14 and Figs. 9 and 10. The proportion of pathological $\mathrm{T} 1$ (pT1; mucosal or submucosal) cancer was $51.2 \%$. The 5 YSR of this population was $90.8 \%$, and the primary cause of death was not cancer recurrence $(n=96)$, but other diseases $(n=207)$. 
Table 25 Survival outcomes by operative procedures

\begin{tabular}{|c|c|c|c|c|c|c|c|c|c|c|c|c|c|c|c|c|c|c|}
\hline & \multirow{2}{*}{$\begin{array}{l}\text { No. of } \\
\text { patients }\end{array}$} & \multicolumn{5}{|c|}{ Postoperative survival rate (\%) } & \multirow{2}{*}{$\begin{array}{l}\text { SE of } \\
5 Y S R\end{array}$} & \multirow[t]{2}{*}{$\mathrm{DD}$} & \multirow{2}{*}{$\begin{array}{l}\text { Lost to } \\
\text { follow } \\
\text { up }\end{array}$} & \multirow[t]{2}{*}{ Alive } & \multicolumn{8}{|c|}{ Main cause of death } \\
\hline & & 1 year & 2 year & 3 year & 4 year & 5 year & & & & & $\mathrm{L}$ & $P$ & $\mathrm{H}$ & M & $\mathrm{R}$ & $\mathrm{OC}$ & OD & UK \\
\hline $\begin{array}{l}\text { Distal } \\
\text { gastrectomy }\end{array}$ & 6684 & 91.6 & 85.5 & 81.6 & 79.1 & 77.2 & 0.5 & 33 & 1173 & 4096 & 133 & 412 & 191 & 75 & 129 & 90 & 267 & 118 \\
\hline $\begin{array}{l}\text { Total } \\
\text { gastrectomy }\end{array}$ & 3377 & 80.0 & 67.5 & 60.6 & 56.1 & 53.7 & 0.9 & 25 & 512 & 1427 & 124 & 612 & 154 & 75 & 155 & 32 & 179 & 107 \\
\hline $\begin{array}{l}\text { Proximal } \\
\text { gastrectomy }\end{array}$ & 446 & 95.2 & 90.0 & 88.3 & 84.3 & 82.3 & 1.9 & 1 & 60 & 312 & 4 & 9 & 6 & 11 & 6 & 9 & 21 & 8 \\
\hline $\begin{array}{l}\text { Pylorus- } \\
\text { preserving }\end{array}$ & 277 & 96.7 & 95.2 & 94.4 & 92.0 & 90.4 & 1.8 & 2 & 32 & 220 & 1 & 2 & 3 & 0 & 2 & 5 & 6 & \\
\hline $\begin{array}{l}\text { Local excision/ } \\
\text { segmental } \\
\text { resection }\end{array}$ & 339 & 95.1 & 94.1 & 89.1 & 84.9 & 82.7 & 2.2 & 2 & 69 & 218 & 4 & 4 & 2 & 0 & 5 & 10 & 20 & \\
\hline $\begin{array}{l}\text { Mucosal } \\
\text { resection }\end{array}$ & 138 & 94.4 & 89.5 & 84.3 & 80.8 & 78.0 & 3.8 & 0 & 31 & 81 & 1 & 1 & 1 & 0 & 1 & 9 & 8 & \\
\hline
\end{tabular}

Table 26 Survival outcomes by lymph node dissection (D)

\begin{tabular}{|c|c|c|c|c|c|c|c|c|c|c|c|c|c|c|c|c|c|c|}
\hline & \multirow{2}{*}{$\begin{array}{l}\text { No. of } \\
\text { patients }\end{array}$} & \multicolumn{5}{|c|}{ Postoperative survival rate $(\%)$} & \multirow{2}{*}{$\begin{array}{l}\text { SE of } \\
5 Y S R\end{array}$} & \multirow[t]{2}{*}{ DD } & \multirow{2}{*}{$\begin{array}{l}\text { Lost to } \\
\text { follow } \\
\text { up }\end{array}$} & \multirow[t]{2}{*}{ Alive } & \multicolumn{8}{|c|}{ Main cause of death } \\
\hline & & 1 year & 2 year & 3 year & 4 year & 5 year & & & & & $\mathrm{L}$ & $\mathrm{P}$ & $\mathrm{H}$ & M & $\mathrm{R}$ & $\mathrm{OC}$ & OD & UK \\
\hline D0 & 812 & 79.1 & 72.7 & 69.2 & 65.1 & 63.7 & 1.8 & 8 & 153 & 394 & 17 & 85 & 25 & 4 & 30 & 28 & 52 & 24 \\
\hline D1 & 2371 & 85.1 & 76.9 & 72.9 & 70.4 & 68.3 & 1.0 & 19 & 340 & 1326 & 48 & 236 & 83 & 31 & 74 & 46 & 137 & 50 \\
\hline $\mathrm{D} 1+\alpha$ & 1368 & 91.3 & 85.8 & 82.2 & 79.6 & 77.5 & 1.2 & 5 & 292 & 799 & 26 & 69 & 40 & 15 & 28 & 17 & 68 & 14 \\
\hline $\mathrm{D} 1+\beta$ & 605 & 94.8 & 90.7 & 87.2 & 84.9 & 83.5 & 1.6 & 2 & 122 & 391 & 5 & 25 & 10 & 5 & 6 & 5 & 26 & 10 \\
\hline D2 & 5403 & 90.7 & 82.8 & 77.5 & 74.0 & 71.8 & 0.6 & 28 & 840 & 3147 & 134 & 523 & 166 & 81 & 142 & 53 & 183 & 134 \\
\hline D3 & 391 & 78.9 & 62.7 & 54.6 & 50.5 & 46.8 & 2.6 & 0 & 30 & 161 & 30 & 82 & 23 & 18 & 15 & 2 & 20 & 10 \\
\hline
\end{tabular}

$\alpha$, Lymph node No. 7 irrespective of the location of lesions, and additionally No. 8a in patients with lesions located in the lower third of the stomach; $\beta$, Lymph nodes No. 7, 8a, 9

Table 27 Survival outcomes by involvement of the resection margins

\begin{tabular}{|c|c|c|c|c|c|c|c|c|c|c|c|c|c|c|c|c|c|c|}
\hline & \multirow{2}{*}{$\begin{array}{l}\text { No. of } \\
\text { patients }\end{array}$} & \multicolumn{5}{|c|}{ Postoperative survival rate $(\%)$} & \multirow{2}{*}{$\begin{array}{l}\text { SE of } \\
5 Y S R\end{array}$} & \multirow[t]{2}{*}{ DD } & \multirow{2}{*}{$\begin{array}{l}\text { Lost to } \\
\text { follow } \\
\text { up }\end{array}$} & \multirow[t]{2}{*}{ Alive } & \multicolumn{8}{|c|}{ Main cause of death } \\
\hline & & 1 year & 2 year & 3 year & 4 year & 5 year & & & & & $\mathrm{L}$ & $P$ & $\mathrm{H}$ & M & $\mathrm{R}$ & $\mathrm{OC}$ & OD & UK \\
\hline $\begin{array}{l}\mathrm{PM}- \\
\text { and } \\
\mathrm{DM}-\end{array}$ & 10550 & 89.5 & 82.3 & 77.7 & 74.6 & 72.5 & 0.5 & 56 & 1784 & 6086 & 232 & 881 & 338 & 136 & 258 & 143 & 466 & 226 \\
\hline $\begin{array}{l}\mathrm{PM}+ \\
\text { and/ } \\
\text { or } \\
\mathrm{DM}+\end{array}$ & 332 & 58.5 & 39.4 & 32.2 & 24.5 & 22.3 & 2.4 & 6 & 34 & 59 & 22 & 119 & 12 & 19 & 31 & 5 & 20 & 11 \\
\hline
\end{tabular}

$P M$ proximal margin, $D M$ distal margin

Peritoneal washing cytology (CY) was carried out for 3481 of 5857 patients with T2, T3, and T4 cancer (59.4\%). The 5YSR of cytology-positive patients (CY1) was $12.3 \%$, which corresponded with that of the patients with peritoneal metastasis (P1) (Tables 17, 18).

The 5YSRs of the patients stratified by the JGCA staging system were $91.9 \%$ for stage IA, $85.1 \%$ for stage
IB, $73.1 \%$ for stage II, $51.0 \%$ for stage IIIA, $33.4 \%$ for stage IIIB, and $15.8 \%$ for stage IV. These JGCA 5YSRs seemed to correlate well with the TNM 5YSRs (Tables 20, 21, 22, 23; Figs. 12, 13).

In regard to the operative procedure, the proportion of patients who underwent laparoscopic gastrectomy was $3.6 \%$, and their 5YSR was $97.4 \%$. Laparoscopic surgery 
Table 28 Survival outcomes by curative potential of gastric resection

\begin{tabular}{|c|c|c|c|c|c|c|c|c|c|c|c|c|c|c|c|c|c|c|}
\hline & \multirow{2}{*}{$\begin{array}{l}\text { No. of } \\
\text { patients }\end{array}$} & \multicolumn{5}{|c|}{ Postoperative survival rate $(\%)$} & \multirow{2}{*}{$\begin{array}{l}\text { SE of } \\
5 Y S R\end{array}$} & \multirow[t]{2}{*}{ DD } & \multirow{2}{*}{$\begin{array}{l}\text { Lost to } \\
\text { follow up }\end{array}$} & \multirow[t]{2}{*}{ Alive } & \multicolumn{8}{|c|}{ Main cause of death } \\
\hline & & 1 year & 2 year & 3 year & 4 year & 5 year & & & & & $\mathrm{L}$ & $\mathrm{P}$ & $\mathrm{H}$ & M & $\mathrm{R}$ & $\mathrm{OC}$ & OD & UK \\
\hline Resection A & 7038 & 97.5 & 94.9 & 92.5 & 90.4 & 88.7 & 0.4 & 20 & 1309 & 5006 & 41 & 72 & 52 & 31 & 49 & 108 & 271 & 99 \\
\hline Resection B & 2593 & 85.0 & 70.7 & 62.1 & 56.3 & 53.1 & 1.0 & 20 & 364 & 1108 & 121 & 380 & 151 & 72 & 119 & 31 & 157 & 90 \\
\hline Resection C & 1420 & 50.3 & 28.7 & 19.7 & 15.5 & 13.4 & 1.0 & 22 & 145 & 145 & 98 & 567 & 152 & 55 & 128 & 10 & 65 & 55 \\
\hline
\end{tabular}

Resection A, no residual disease with high probability of cure satisfying all of the following conditions: T1 or T2; N0 treated by D1, 2, 3 resection or N1 treated by D2, 3 resection; M0, P0, H0, CY0, and proximal and distal margins $>10 \mathrm{~mm}$; Resection $B$, no residual disease but not fulfilling criteria for "Resection A"; Resection C, definite residual disease

Fig. 3 Kaplan-Meier survival for all 12004 patients with primary gastric cancer. $5 Y S R$ 5-year survival rate
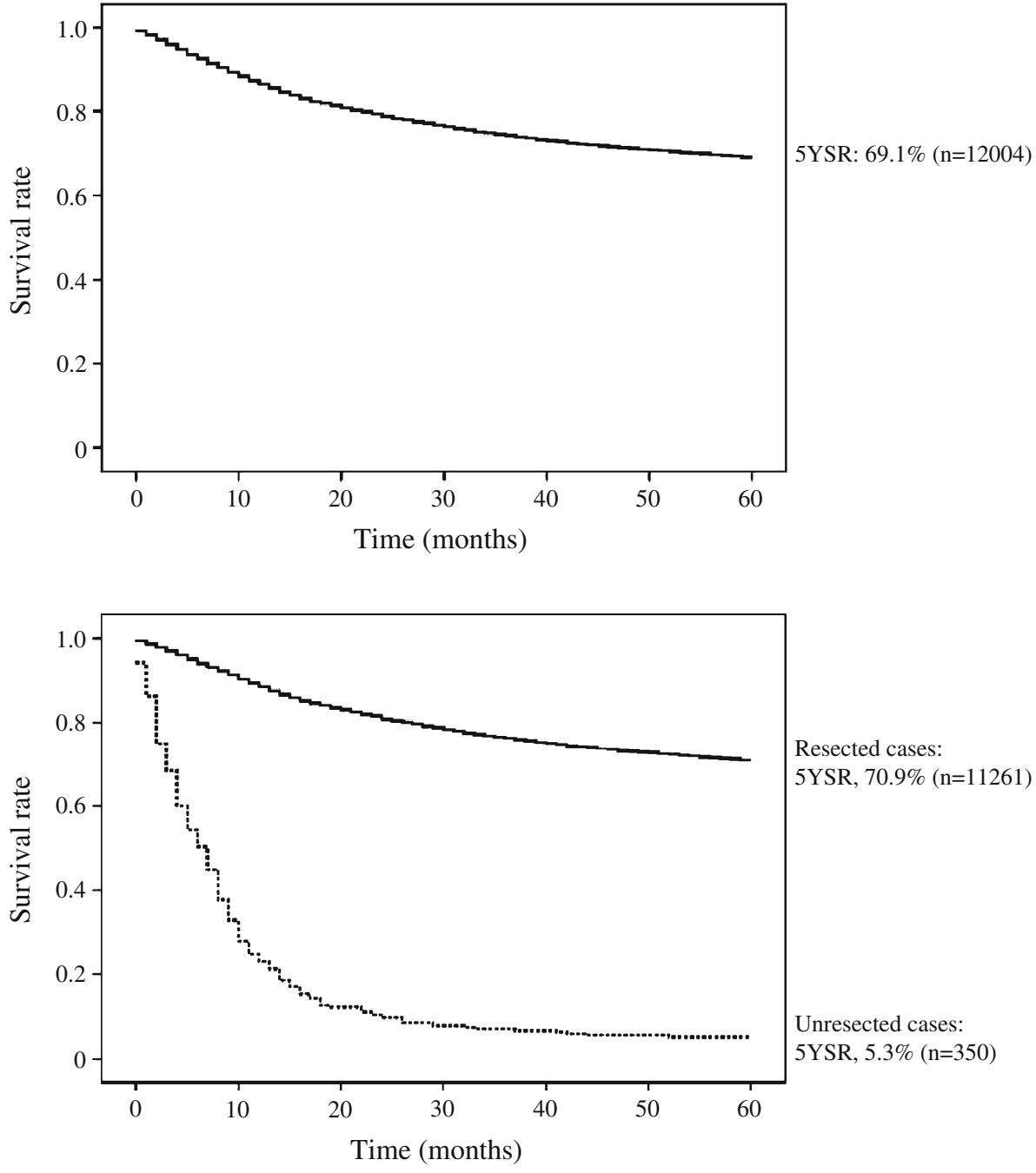

Fig. 4 Kaplan-Meier survival for resected cases and unresected cases was carried out mainly in patients with early gastric cancer. Only $1.0 \%$ of the patients were treated by thoraco-laparotomy, and their 5YSR was $40.7 \%$. Thoraco-laparotomy was carried out in patients with gastric cardia cancer invading the esophagus (Table 24). Thirty percent of the patients underwent total gastrectomy, and their 5YSR was $53.7 \%$. The proportion of patients treated by modified surgery such as proximal gastrectomy, pylorus-preserving gastrectomy, segmental gastrectomy, and local resection was $9.4 \%$ (Table 25 ). D0, D1, D1+ $\alpha$, and D1+ $\beta$ dissections were carried out in $7.4,21.7,12.5$, and $5.5 \%$ of the patients, respectively. According to the JGCA gastric cancer treatment guidelines [7, 8], D1+ $\alpha$ dissection with modified gastrectomy was indicated for T1(M)N0 tumors and T1(SM)N0 differentiated tumors $<1.5 \mathrm{~cm}$ in diameter, while $\mathrm{D} 1+\beta$ dissection with modified gastrectomy was indicated for T1(SM)N0 undifferentiated tumors, T1(SM)N0 differentiated tumors larger than $1.6 \mathrm{~cm}$, 
Fig. 5 Kaplan-Meier survival of the resected cases stratified by sex

Fig. 6 Kaplan-Meier survival of the resected cases stratified by age

Fig. 7 Kaplan-Meier survival of the resected cases stratified by tumor location. $W$ whole stomach, $M$ middle third, $L$ lower third, $U$ upper third of stomach
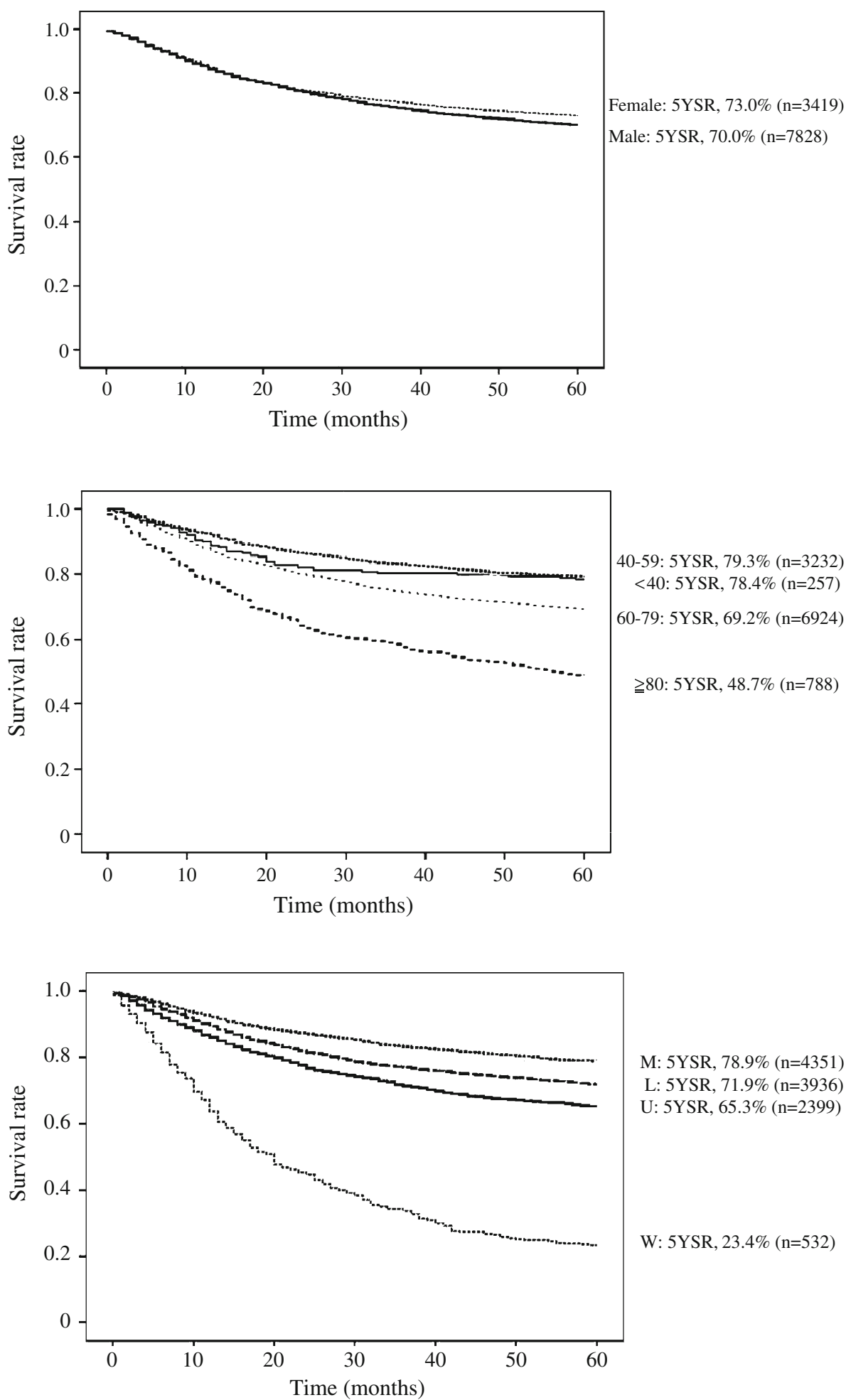

T1(M)N1 tumors, and T1(SM)N1 tumors $<2.0 \mathrm{~cm}$. D0 and D1 dissections were carried out mainly in patients with non-curative factors or poor surgical risks. D2 lymph node dissection was carried out in $49.3 \%$ of the patients and the risk of direct death in those with D2 gastrectomy was $0.5 \%$ (28/5403; Table 26).
The curative potential of gastric resection was an important prognostic factor. The proportion of patients with a high probability of cure (resection A) was $63.7 \%$, and their 5YSR was $88.7 \%$. On the other hand, the proportion of patients with definite residual tumor (resection C) was $12.8 \%$, and their 5YSR was $13.4 \%$ (Table 28; Fig. 14). 
Fig. 8 Kaplan-Meier survival of the resected cases stratified by macroscopic type

Fig. 9 Kaplan-Meier survival of the resected cases stratified by depth of tumor invasion. $M$ mucosa or muscuralis mucosa, $S M$ submucosa, $M P$ muscularis propria, $S S$ subserosal, $S E$ serosa, $S I$ adjacent structures
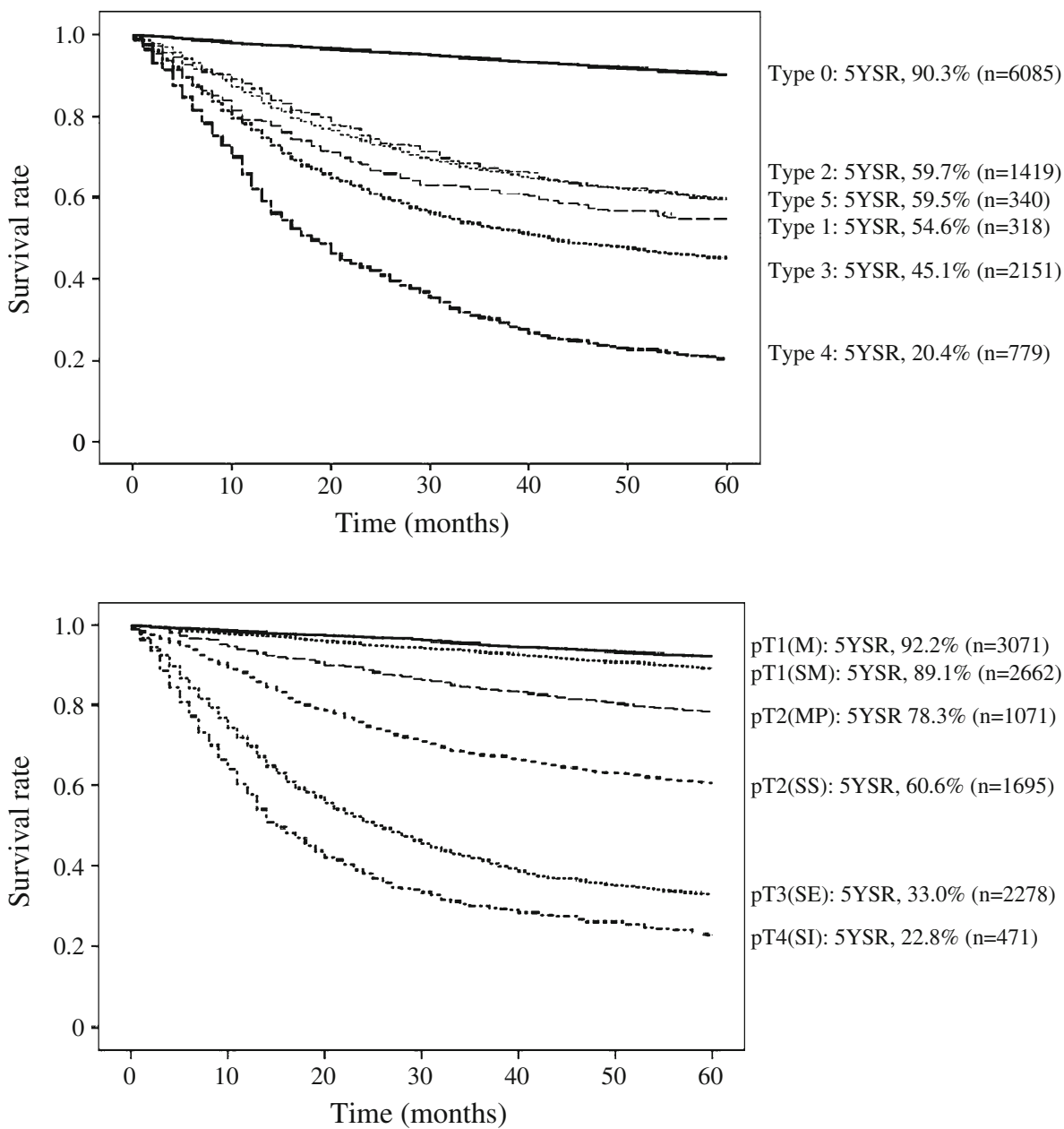

Fig. 10 Kaplan-Meier survival of the resected cases stratified by $\mathrm{pT}$ classification

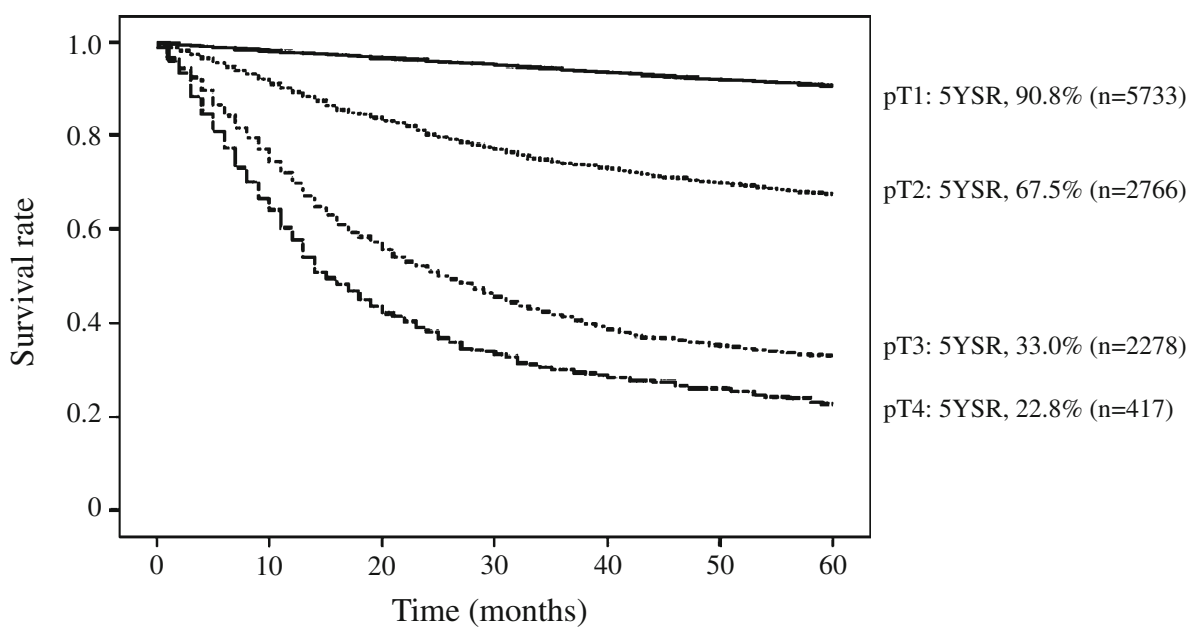

\section{Discussion}

The data presented in this report were collected from 187 hospitals in Japan. The number of new patients who were diagnosed with gastric cancer in 2001 was estimated to be 107726 [9]. Accordingly, the 11261 patients registered by this program corresponded to approximately $10 \%$ of the population affected by gastric cancer in Japan. Even though these patients may not represent the average features of gastric cancer, this article is considered to be the largest report for the past 10 years clarifying the trends of gastric cancer. 
Fig. 11 Kaplan-Meier survival of the resected cases stratified by lymph node metastasis

Fig. 12 Kaplan-Meier survival of the resected cases stratified by Japanese Gastric Cancer Association (JGCA) stage

Fig. 13 Kaplan-Meier survival of the resected cases stratified by TNM stage
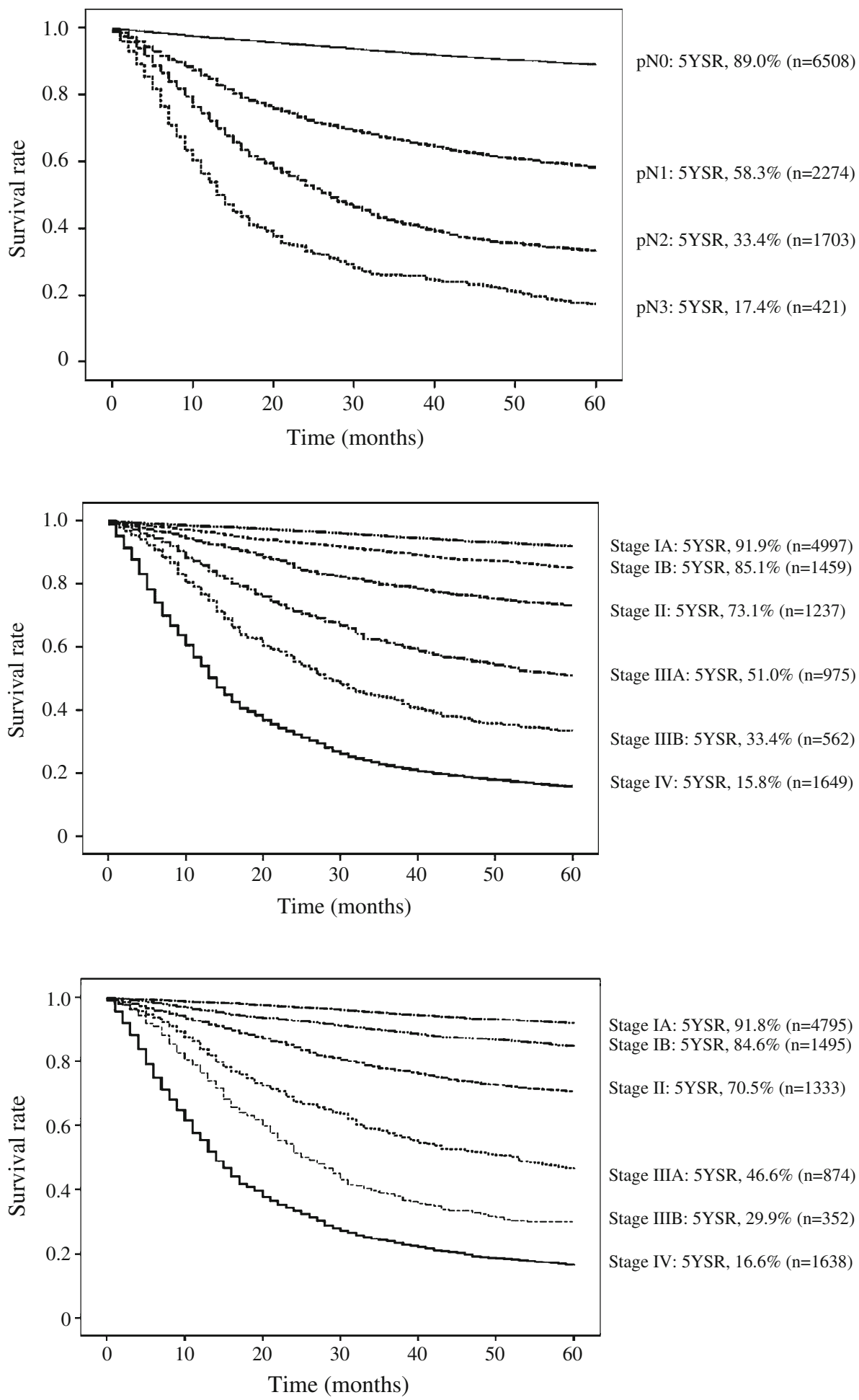

The reliability of the results in this report depends on the quality of data accumulated in the JGCA database. As the algorithms of the JGCA staging system were rather complicated, the error checking system on the data entry screen did not work perfectly. In several categories, such as lymph node metastasis $(\mathrm{N})$, the JGCA code could not convert to the TNM code automatically. A few "bugs" in the software were revealed just after we had analyzed thousands of data records. Therefore, the registration committee had to make great efforts to cleanse and validate the raw data sent to the data center from participating hospitals.

As compared with our archived data of 7935 patients treated in 1991 [1], though the proportions of each stage were similar, the direct death rate had significantly 
Fig. 14 Kaplan-Meier survival of the resected cases stratified by curative potential of gastric resection. Resection A, no residual disease with high probability of cure satisfying all of the following conditions: $\mathrm{T} 1$ or T2; N0 treated by D1, 2, 3 resection or $\mathrm{N} 1$ treated by $\mathrm{D} 2,3$ resection; $\mathrm{M} 0, \mathrm{P} 0, \mathrm{H} 0, \mathrm{CYO}$, and proximal and distal margins $>10 \mathrm{~mm}$; Resection $B$, no residual disease but not fulfilling criteria for "Resection A"; Resection C, definite residual disease

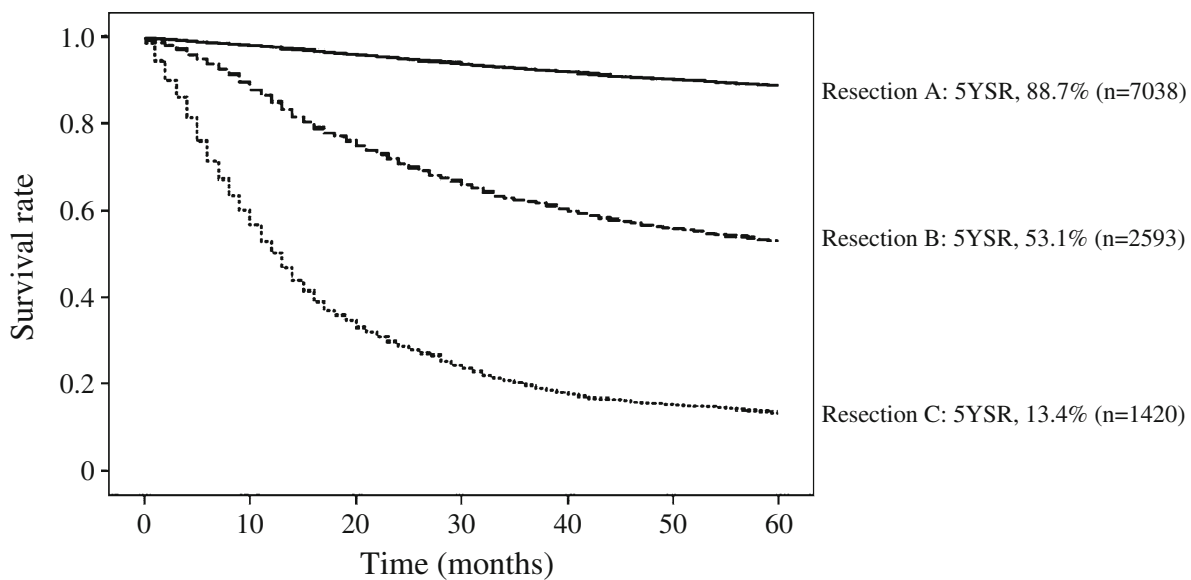

Table 29 Five-year follow-up rates stratified by TNM stage

\begin{tabular}{lccc}
\hline & No. of patients & Lost to follow up & FUR (\%) \\
\hline Stage I & 6290 & 1241 & 80.3 \\
Stage II & 1333 & 201 & 84.9 \\
Stage III & 1226 & 173 & 85.9 \\
Stage IV & 1638 & 157 & 90.4 \\
Total & 10487 & 1772 & 83.1 \\
\hline
\end{tabular}

FUR 5-year follow-up rate

improved, dropping from 1.0 to $0.6 \%(P<0.001)$; the proportion of patients aged more than 80 years old had increased, from 4.5 to $7.0 \%(P<0.001)$; and the 5YSR of stage IV had improved, from 9.0 to $15.8 \%(P<0.05)$. These data suggest that, in this decade, the treatment results may have improved in patients with advanced disease and in older patients.

However, these data were retrospectively collected, 7 years after surgery. We had legal difficulties in registering personal information, which was essential for longterm and prospective follow-up. The overall follow-up rate in our program was $83.5 \%$, as already mentioned. A lower follow-up rate is generally considered to show misleading results of higher survival rates in patients with advanced disease. The Japanese Association of Clinical Cancer Centers (consisting of 25 cancer center hospitals) has reported that their follow-up rate was $98.5 \%$, and the 5YSRs of 9980 patients who underwent surgery from 1997 to 2000 were $90.4 \%$ for TNM stage I, $67.8 \%$ for stage II, $43.3 \%$ for stage III, and $9.3 \%$ for stage IV [10]. On the other hand, our 5YSR in stage IV patients was $16.6 \%$ (Table 23). We might have overestimated our 5YSR in stage IV patients, but we found that the follow-up rate increased as the stage advanced; the follow-up rate of stage IV patients was $90.4 \%$ (Table 29). Of the 187 participating hospitals, 114 hospitals achieved high follow-up rates of $90 \%$ or more for stage IV patients. Therefore, the 5-year
Table 30 Follow-up rates and survival rates stratified by TNM stage in 187 participating hospitals and 114 selected hospitals

\begin{tabular}{|c|c|c|c|c|c|c|}
\hline \multirow{2}{*}{$\begin{array}{l}\text { TNM } \\
\text { stage }\end{array}$} & \multicolumn{3}{|c|}{187 Participating hospitals } & \multicolumn{3}{|c|}{114 Selected hospitals } \\
\hline & $\begin{array}{l}\text { No. of } \\
\text { patients }\end{array}$ & $\begin{array}{l}\text { FUR } \\
(\%)\end{array}$ & $\begin{array}{l}5 Y S R \\
(\%)\end{array}$ & $\begin{array}{l}\text { No. of } \\
\text { patients }\end{array}$ & $\begin{array}{l}\text { FUR } \\
(\%)\end{array}$ & $\begin{array}{l}5 Y S R \\
(\%)\end{array}$ \\
\hline Stage IA & 4795 & 80.2 & 91.8 & 3401 & 84.0 & 91.3 \\
\hline Stage IB & 1495 & 80.6 & 84.6 & 1000 & 84.2 & 82.5 \\
\hline Stage II & 1333 & 84.9 & 70.5 & 938 & 89.6 & 70.3 \\
\hline Stage IIIA & 874 & 84.7 & 46.6 & 608 & 93.1 & 45.2 \\
\hline Stage IIIB & 352 & 88.9 & 29.9 & 243 & 93.8 & 30.8 \\
\hline Stage IV & 1638 & 90.4 & 16.6 & 1196 & 97.7 & 15.9 \\
\hline
\end{tabular}

The 114 hospitals were selected on the criterion of achieving high follow-up rate of $90 \%$ or more for stage IV patients

follow-up rates and 5YSRs in these 114 hospitals were calculated for reference. The mean follow-up rate for stage IV patients in these 114 selected hospitals was $97.7 \%$ and their 5YSR was $15.9 \%$ (Table 30). These data suggest that the lower follow-up rate in our program may not have serious effects on the 5YSRs. Although the correlation between follow-up rate and survival rate is complicated, we need to greatly improve our follow-up system to evaluate our survival rates more accurately.

This is the first nationwide report in which the JGCA refers to peritoneal washing cytology (CY). CY was conducted in $3481(59.4 \%)$ of 5857 patients with T2, T3, or T4 cancer. The 5YSR of CY-positive (CY1) patients was $12.3 \%$ and their 5YSR was as poor as that of patients with peritoneal metastasis (P1; 12.4\%). Although CY was not carried out commonly in 2001, it was regarded as a significant and independent prognostic factor.

The JGCA restarted a nationwide registration program after an inactive period of 10 years. The most urgent priority of this program was to report detailed 5YSRs in patients who had received a gastrectomy. Therefore, the structure of the database was required to be simple and the 
number of registration items was kept to a minimum. We are now planning to register more items concerning remnant gastric cancer, chemotherapy, and endoscopic submucosal dissection by upgrading the data entry software. We will continue our efforts to collect qualified data annually.

Acknowledgments The JGCA Registration Committee appreciates very much the great effort of member hospitals in registering accurate and detailed data for this project. We also wish to thank Ms. Yoshimi Sugamura, Niigata University Medical and Dental Hospital, for her valuable assistance.

Open Access This article is distributed under the terms of the Creative Commons Attribution Noncommercial License which permits any noncommercial use, distribution, and reproduction in any medium, provided the original author(s) and source are credited.

\section{Appendix: Member hospitals}

Data of gastric cancer patients in this report were collected from the surgical or gastrointestinal departments of the following 187 hospitals (in alphabetical order).

Aichi Cancer Center Aichi Hospital, Aichi Cancer Center Hospital, Akashi Municipal Hospital, Aomori City Hospital, Asahikawa Medical University, Cancer Institute Hospital, Chiba Cancer Center, Chiba University Hospital, Dokkyo Medical University, Ebina General Hospital, Fuchu Hospital, Fujita Health University (Banbuntane Houtokukai Hospital), Fujita Health University Hospital, Fukui Red Cross Hospital, Fukui Saiseikai Hospital, Fukuoka University Chikushi Hospital, Fukuoka University Hospital, Fukushima Medical University Hospital, Gunma Prefectural Cancer Center, Gunma University Graduate School of Medicine (Department of General Surgical Science), Gunma University Graduate School of Medicine (Department of Thoracic Visceral Organ Surgery), Hachioji Digestive Disease Hospital, Hakodate Goryoukaku Hospital, Hakodate Municipal Hospital, Hamamatsu University School of Medicine, Hamanomachi Hospital, Health Insurance Naruto Hospital, Higashiosaka City General Hospital, Himeji Central Hospital, Hirakata City Hospital, Hiroshima City Hospital, Hiroshima Prefectural Hospital, Hiroshima University Hospital, Hitachi General Hospital, Hoshigaoka Koseinenkin Hospital, Hyogo Cancer Center, Hyogo Prefectural Nishinomiya Hospital, Ibaraki Prefectural Central Hospital, Ibaraki Seinan Medical Center Hospital, Ichinomiya Municipal Hospital, Imamura Hospital, Iwate Prefectural Central Hospital, Iwate Prefectural Isawa Hospital, Iwate Prefectural Kamaishi Hospital, JA Hiroshima Kouseiren Hiroshima General Hospital, Jichi Medical University Hospital, Jikei University School of Medicine (Aoto Hospital), Kagawa University Hospital,
Kakogawa Municipal Hospital, Kanagawa Cancer Center, Kanazawa Medical University Hospital, Kawasaki Medical School Hospital, Kawasaki Municipal Hospital, Keio University School of Medicine, Keiyukai Sapporo Hospital, Kimitsu Chuo Hospital, Kinki Central Hospital, Kinki University School of Medicine (Nara Hospital), Kiryu Kosei General Hospital, Kitakyushu Municipal Medical Center, Kitasato Institutional Hospital, Kitasato University East Hospital, Kobe City Medical Center General Hospital, Kobe University Hospital, Koga General Hospital, Kokura Memorial Hospital, Kouchi Medical School Hospital, Kumamoto Regional Medical Center, Kumamoto University Hospital, Kurashiki Central Hospital, Kurobe City Hospital, Kushiro Rosai Hospital, Kyorin University Hospital, Kyoto Prefectural University of Medicine, Kyoto Prefectural Yosanoumi Hospital, Kyoto University Hospital, Kyushu University Hospital, Matsue City Hospital, Matsushita Memorial Hospital, Matsuyama Shimin Hospital, Minami Tohoku Hospital, Misawa City Hospital, Mitoyo General Hospital, Mitsui Memorial Hospital, Miyagi Cancer Center, Muroran General Hospital, Musashino Red Cross Hospital, Nagahama City Hospital, Nagano Municipal Hospital, Nagaoka Chuo General Hospital, Nagoya City University Hospital, Nagoya University Hospital, Nanpuh Hospital, Nara Medical University Hospital, Narita Red Cross Hospital, National Defense Medical College, National Kyushu Cancer Center, NHO Ciba Medical Center, NHO Ibusuki Hospital, NHO Kasumigaura Medical Center, NHO Kobe Medical Center, NHO Nagasaki Medical Center, NHO Osaka Medical Center, NHO Sendai Medical Center, NHO Shikoku Cancer Center, NHO Tokyo Medical Center, Niigata Cancer Center Hospital, Niigata Prefectural Shibata Hospital, Niigata University Medical and Dental Hospital, Nippon Medical School Chiba Hokusoh Hospital, Nippon Medical School Musashikosugi Hospital, Nippon Medical School, NTT West Osaka Hospital, Obihiro Tokushukai Hospital, Oita Red Cross Hospital, Oita University Hospital, Okayama Saiseikai General Hospital, Okayama University Hospital, Okitama Public General Hospital, Onomichi Municipal Hospital, Osaka City University Hospital, Osaka General Medical Center, Osaka Kouseinenkin Hospital, Osaka Medical Center for Cancer and Cardiovascular Diseases, Osaka Red Cross Hospital, Otsu Municipal Hospital, Otsu Red Cross Hospital, Ryukyu University School of Medicine, Saga University Hospital, Sagamihara Kyodo Hospital, Saiseikai Fukuoka General Hospital, Saiseikai Maebashi Hospital, Saiseikai Niigata Daini Hospital, Saiseikai Noe Hospital, Saitama Medical Center, Saitama Red Cross Hospital, Saitama Social Insurance Hospital, Sakai Municipal Hospital, Saku Central Hospital, Sapporo Social Insurance General Hospital, Sayama Hospital, Seirei Hamamatsu General Hospital, Seirei Mikatahara General 
Hospital, Self-defense Forces Central Hospital, Sendai Open Hospital, Sendai Red Cross Hospital, Shiga Medical Center for Adults, Shiga University of Medical Science, Showa General Hospital, Showa University Toyosu Hospital, Social Insurance Central General Hospital, Social Insurance Kinan Hospital, St. Luke's International Hospital, Suita Municipal Hospital, Surugadai Nihon University Hospital, Tochigi Cancer Center, Toho University Ohashi Medical Center, Tokushima Municipal Hospital, Tokushima University Hospital, Tokyo Dental College Ichikawa General Hospital, Tokyo Medical University, Tokyo Metropolitan Bokutoh Hospital, Tokyo Metropolitan Cancer and Infectious Disease Center Komagome Hospital, Tokyo Metropolitan Police Hospital, Tokyo Women's Medical University (Institute of Gastroenterology), Tokyo Women's Medical University Hospital (Department of Surgery 2), Tokyo Women's Medical University Medical Center East, Tonami General Hospital, Toranomon Hospital, Tottori University Hospital, Toyama University Hospital, Tsuchiura Kyodo General Hospital, Tsuruoka Municipal Shonai Hospital, University of Fukui Hospital, University of Miyazaki Hospital, University of Tokyo Hospital, University of Yamanashi Hospital, Wakayama Medical University, Yamagata Prefectural Central Hospital, Yamagata Prefectural Kahoku Hospital, Yamagata University Hospital, Yamaguchi Rousai Hospital, Yamanashi Prefectural Central Hospital, Yao Municipal Hospital, Yodogawa Christian Hospital, Yokohama City University Medical Center, Yuai Memorial Hospital.

\section{References}

1. Maruyama K, Kaminishi M, Hayashi K, Isobe Y, Honda I, Katai H, et al. Gastric cancer treated in 1991 in Japan: data analysis of nationwide registry. Gastric Cancer. 2006;9:21-66.

2. Nashimoto A, Akazawa K, Isobe Y, Miyashiro I, Oda I, Kaminishi $\mathrm{M}$, et al. The present state and problems of gastric cancer treatment from the view points of nationwide registry. Jpn J Cancer Clin. 2009;55:713-8 (in Japanese).

3. Isobe Y, Nashimoto A, Akazawa K, Hayashi K, Miyashiro I, Oda $\mathrm{I}$, et al. Problems and future perspectives on the nationwide registry of gastric cancer. Gekachiryo. 2010;102:358-64 (in Japanese).

4. Japanese Gastric Cancer Association. Japanese classification of gastric carcinoma. 13 ed. Tokyo: Kanehara; 1999 (in Japanese).

5. Japanese Gastric Cancer Association. Japanese classification of gastric carcinoma-2nd English edition. Gastric Cancer. 1998;1:10-24.

6. International Union Against Cancer. Sobin LH, Wittekind C, editors. TNM classification of malignant tumors. 5th ed. New York: WILEY-LISS; 1997.

7. Japanese Gastric Cancer Association. Gastric cancer treatment guidelines for doctors' reference. Tokyo: Kanehara; 2001 (in Japanese).

8. Japanese Gastric Cancer Association. Introduction to JGCA gastric cancer treatment guidelines. http://www.jgca.jp/PDFfiles/ E-guideline.PDF (2001).

9. Marugame T, Matsuda T, Kamo K, Katanoda K, Ajiki W, Sobue $\mathrm{T}$, et al. Cancer incidence and incidence rates in Japan in 2001 based on the data from 10 population-based cancer registries. Jpn J Clin Oncol. 2007;37:884-91.

10. Survival rate in the member hospitals of the Association of Clinical Cancer Centers (diagnosed in 1997-2000). In: Kato H, Sobue T, Katanoda K, Saito Y, Tukuma H, Saruki N, et al., editors. Cancer statistics in Japan-2008. Tokyo: Foundation for Promotion of Cancer Research; 2009. p. 81. 